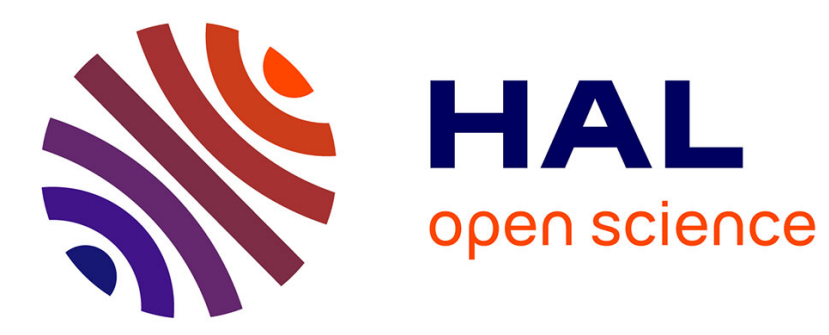

\title{
Robust Noncooperative Spectrum Sharing Game in Underwater Acoustic Interference Channels
}

\author{
Antony Pottier, François-Xavier Socheleau, Christophe Laot
}

\section{To cite this version:}

Antony Pottier, François-Xavier Socheleau, Christophe Laot. Robust Noncooperative Spectrum Sharing Game in Underwater Acoustic Interference Channels. IEEE Journal of Oceanic Engineering, 2017, 42 (4), pp.1019-1034. 10.1109/JOE.2017.2731060 . hal-01630275

\section{HAL Id: hal-01630275 \\ https://hal.science/hal-01630275}

Submitted on 7 Nov 2017

HAL is a multi-disciplinary open access archive for the deposit and dissemination of scientific research documents, whether they are published or not. The documents may come from teaching and research institutions in France or abroad, or from public or private research centers.
L'archive ouverte pluridisciplinaire HAL, est destinée au dépôt et à la diffusion de documents scientifiques de niveau recherche, publiés ou non, émanant des établissements d'enseignement et de recherche français ou étrangers, des laboratoires publics ou privés. 


\title{
Robust Noncooperative Spectrum Sharing \\ Game in Underwater Acoustic Interference
}

\section{Channels}

\author{
Antony Pottier, François-Xavier Socheleau, and Christophe Laot
}

A. Pottier, F-X. Socheleau and C. Laot are with IMT Atlantique, Lab-STICC, Univ. Bretagne Loire, 29238 Brest, France (email: \{antony.pottier, fx.socheleau, christophe.laot\} imt-atlantique.fr). Part of this work has been presented at the $17^{\text {th }}$ IEEE Workshop on Signal Processing Advances in Wireless Communications (SPAWC), July 2016 [1], and at the $3^{\text {rd }}$ Underwater Communications and Networking Conference (UComms), September 2016 [2].

\begin{abstract}
This paper focuses on underwater acoustic (UA) communications and proposes a decentralized spectrum sharing method for noncooperative orthogonal frequency-division multiplexing systems in interference channels. The problem is formulated as a noncooperative game where the players are UA communication systems aiming at finding the power allocation on subcarriers that maximizes a utility function related to their information rate. Realistic assumptions regarding the UA context are formulated. Frequency-selective and randomly time-varying channels are considered. Each system is constrained in average power and adapts its power allocation strategy only with local knowledge of its channel statistics and noise plus interference power spectral density. This knowledge is obtained through a feedback link from the receiver. Estimation errors on the channel statistics are taken into account, thanks to a robust reformulation of the game. We show that an efficient decentralized spectrum sharing can be achieved when all players use a water-filling strategy against each other iteratively. Simulations results are obtained on synthetic but realistic channels. In configurations where the UA communication systems are in close areas, significant increases of spectral efficiencies can be expected compared to the conventional uniform power allocation. Results on channels sounded at sea support our conclusions.
\end{abstract}




\section{INTRODUCTION}

The underwater acoustic (UA) propagation channel is shared by several heterogeneous entities that use acoustic waves to communicate, navigate or infer information about the marine environment. Although initiatives have been taken [3], the time and frequency activity of UA devices is yet not restricted by regulatory bodies. Moreover, the usable bandwidth decreases with the transmission distance and frequency [4]. Thus, the availability of a physical resource for UA communication systems varies with time, frequency, as well as with the number and location of other active sound sources [5]. In the absence of regulation, the simultaneous anarchical access to the UA channel by several sources can lead to mutual interference that degrades the reliability of communication systems. Several examples of jamming by external sound sources have been reported in the recent literature [6], [7]. As the ongoing and future development of underwater applications is likely to increase the anthropogenic acoustic noise [8], there is an NEed for better coexistence of various sound sources in the UA transmission channel.

A possible approach to improve the reliability of UA communications corrupted by interfering sounds is to mitigate them at the receiver [6], [7], [9]. However, it often requires some prior knowledge of the interfering waveforms [6], [7], or the ability for receivers to decode messages from other users [9]. An alternative strategy is to allow communicating devices to learn autonomously their best transmission strategies thanks to repeated interactions with their environment. This environment being partly constituted by other users. This approach is more flexible as it is a decentralized way to deal with noncooperative UA devices competing for the same resource.

The benefits of adaptive modulations for single-user UA communications have been shown in several recent works [10]-[14]. The joint optimization of modulation orders and frequency allocations under a bit error rate constraint is proposed in [10] for UA OFDM systems. Based on previous work, [11] studied the achievable rates over Rician-fading channels for UA-OFDM communications implementing different practical power allocation strategies. The authors in [12] propose an algorithm that enables the transmitters to adapt their power according to the large scale fading of the channel, with the objective of maintaining a constant signal to noise ratio (SNR) at the receiver side. Other adaptive schemes were proposed more recently in [13], where an OFDM transmitter can switch between several configurations according to a performance metric feedback by its receiver. A decision tree-based adaptive modulation is also proposed in [14].

Advances have been realized in the development of Media Access Control (MAC) protocols for multiuser UA communications, whose objective is to avoid collisions between acoustic signals by allocating 
different codes, time slots or frequency bands to network users. A good survey of these techniques can be found in [15], [16]. To the best of our knowledge, most of the research efforts on resource allocation for multiuser UA communications have focused on homogeneous systems evolving within the same network.

We consider here noncooperative systems as a more general framework to deal with the interference problem in UA communications. By noncooperative, we mean that several UA communication systems are competing for the use of the same physical resource without the ability to exchange their channel knowledge to agree on a fair sharing scheme. Going further, it also means that they may not be aware that other devices are also trying to use this common resource. Nevertheless, by some form of repeated interactions with their surrounding environment, all transmitters should be able to optimize their parameters to avoid situations where none of them is reliable. This optimization can be done on the basis of some metrics on the communication link quality sent back from receivers to their corresponding transmitters.

The study of interactions between several independent decision-makers (or agents) with conflicting interests can be mathematically formalized by game theory. Within this framework, each UA channel user is considered as a player choosing among strategies (e.g., its transmission power or rate, or its coding/modulation scheme) to maximize its own objective function, also called utility function. Solving a game consists in finding a choice of strategies that drives the players to an equilibrium state. These tools have been used successfully in the field of terrestrial communications during the past decade [17], [18]. Nevertheless, the assumptions of known time-invariant channel or known channel realizations are often made in the cited works and references therein. In the UA context, these assumptions can hardly be valid because of random time-varying fading processes typical of UA channels.

In this paper, a formulation of the competitive spectrum sharing problem for UA communication systems as a noncooperative game is proposed, and extends recent works on the topic [1], [2]. Every transmitter/receiver pair is a player who wants to find the power allocation that maximizes a performance metric related to its information rate, under average power constraint. Communications based on OFDM are considered since it is well suited to spectrum sharing issues. The problem is solved according to the Nash Equilibrium (NE) solution [19], which is appropriate to noncooperative setups. This equilibrium is defined as a joint choice of strategies such that none of the agents has an interest to unilaterally deviate from. In the context of this work, it corresponds to a spectrum sharing where every communication link plays its optimal strategy in the sense that it cannot choose another strategy unless it pays the price of a rate loss. In practice the agents involved in the game can learn an equilibrium through repeated interactions with each others [17], [20]. This learning process is actually implemented by iterative 
algorithms, distributed among the agents, which compute a solution of the game.

This study proposes an iterative, distributed spectrum sharing algorithm for noncooperative UA-OFDM communications, where each transmitter/receiver pair can determine its optimal power allocation only on the basis of a local and possibly erroneous knowledge of the interference environment. The proposed method takes explicitly into account realistic constraints imposed by the UA context. A randomly timevarying frequency-selective UA channel is considered. The transmitters update their power allocation using a water-filling strategy based on a statistical channel state information (CSI), which is supposed to be sent back periodically by their corresponding receivers. A robust spectrum sharing strategy is proposed. This approach allows the users in competition to be more conservative about how they use their power budget by favoring their best subchannels. It is experimentally shown that this behavior can be highly beneficial in some situations. More specifically, the robust method is able to drive UA-OFDM systems toward spectrum sharing strategies that are Pareto superior to the NE. These strategies increase utilities reached by at least one channel user, without being detrimental to the others. This improvement in equilibrium efficiency is done without introducing centralized control, coordination or information exchange between users.

The paper is organized as follows. Section II describes the transmission and channel models and states the spectrum sharing problem. Section III defines the set of players with their strategies and utility functions, then formulates the problem as a noncooperative game. A solution based on the Nash Equilibrium is given in Section IV, where it is assumed that UA users have perfect knowledge of their channel and noise plus interference statistics. In Section V, this assumption of perfect knowledge is relaxed. A robust game that takes estimation errors into account is formulated. The proposed spectrum sharing strategies are evaluated by means of simulations on synthetic channels in Section VI. Results on channels sounded at-sea are given in Section VII and confirm the relevance of our approach, before conclusion in Section IX.

Notations: Uppercase and lowercase boldface letters, e.g. A, x, denote matrices and vectors, respectively. The superscript ${ }^{T}$ denotes transposition. The identity matrix of size $N \times N$ is $\mathbf{I}_{N}$. The Hadamard product is denoted by $\odot . \mathcal{L}^{2}$ denotes the vector space of random variables with finite variance. $a$ mod $n$ is the remainder of the Euclidean division of $a$ by $n$. The matrix of size $N \times N$ having the $N$-elements vector $\mathbf{x}$ on its diagonal and zeros elsewhere is denoted by $\operatorname{diag}(\mathbf{x})$. Finally, $\mathbb{E}\{$.$\} denotes expectation.$ 


\section{PRoblem Statement}

An UA interference channel is considered where a finite set $\mathcal{M}=\{1, \cdots, M\}$ of OFDM transmitter/receiver pairs are in competition for the use of the same bandwidth $B$ divided into $N$ subcarriers whose spacing is $\Delta f$. In the following, the transmitter/receiver pairs are called (communication) links, users or players interchangeably. Focusing on noncooperative setups, each receiver treats the interference of unwanted transmitters as additive colored noise. The channel is assumed to be frequency-selective and time-varying. Its coherence bandwidth and coherence time are, respectively, greater than $\Delta f$ and the OFDM block duration $T=T_{s}+T_{g}$, with $T_{s}=1 / \Delta f$ where $T_{g}$ the cyclic prefix duration. For the sake of ease of presentation, the transmission parameters $N, B, \Delta f$, and $T_{g}$ are the same for all users. Having different transmission parameters for each user would not change the spectrum sharing solution proposed in the following.

Let $\mathbf{x}_{i}(n) \in \mathbb{C}^{L}$ be a vector of $L$ zero-mean i.i.d. input symbols sent by the transmitter $i \in \mathcal{M}$ on the $n^{\text {th }}$ subcarrier. For any $i \in \mathcal{M}$ and $n \in\{1, \cdots, N\}$, the symbols $\mathbf{x}_{j \neq i}(n)$ sent by the transmitters $j \in \mathcal{M}, j \neq i$, are independent of $\mathbf{x}_{i}(n)$. Each transmitter $i$ is subject to its own power constraint

$$
\sum_{n=1}^{N} p_{i}(n) \leq P_{i}^{\max },
$$

where $p_{i}(n)$ is the power allocated by $i$ on the $n^{\text {th }}$ subcarrier.

After cyclic prefix removal and discrete Fourier transform, the channel output observed over $L$ OFDM symbols by the receiver $i$ on the $n^{\text {th }}$ subcarrier can be written as

$$
\mathbf{y}_{i}(n) \triangleq \mathbf{h}_{i i}(n) \odot \mathbf{x}_{i}(n)+\sum_{j \neq i} \mathbf{h}_{j i}(n) \odot \mathbf{x}_{j}(n)+\mathbf{w}_{i}(n),
$$

where $\mathbf{w}_{i}(n) \sim \mathcal{C N}\left(0, \sigma_{i}^{2}(n) \mathbf{I}_{L}\right)$ is a Gaussian noise independent of both $\mathbf{x}_{i}(n)$ and $\mathbf{x}_{j \neq i}(n)$, and $\mathbf{h}_{j i}(n) \in$ $\mathbb{C}^{L}$ are $L$ realizations of a random variable $h_{j i}(n)$ modeling the channel between transmitter $j$ and receiver $i$ at the $n^{\text {th }}$ subcarrier. Each player $i$ only has a statistical knowledge of its own channel $h_{i i}(n)$, which is assumed statistically stationary during a block of $L$ symbols. ${ }^{1}$ For any player $i$, nothing is known about $h_{j i}(n)$ or its realizations if $j \neq i$. Doppler effects are assumed to be compensated and it is not assumed that users are synchronized. ${ }^{2}$

In situations where several communication systems are in the same geographical area and try to access simultaneously to the same physical resource, it may be that some or none of them are actually reliable,

\footnotetext{
${ }^{1}$ Block-stationarity is an approximation since, in practice the taps statistics can be slowly time-varying [21], [22].

${ }^{2}$ Intercarrier interference due to OFDM symbols misalignment between the different users is not explicitly taken into account here as, in light of [23], it does not change significantly the problem formulation.
} 
due to a strong interference level. Our goal is to allow noncooperative users to share the available spectrum more efficiently by choosing judiciously the subcarriers on which they allocate their power. This choice must be made such that each link maximizes some performance criterion that depends on the ambient soundscape in which it operates. Automatic adaptation of the transmit power requires that transmitters have some knowledge on their acoustic environment, namely the channel state and perceived interferences. Practically, this can be made possible via a feedback link from their intended receivers. Given that other systems are also accessing the channel, the performance of one particular system depends on the actions taken by the others. The UA-OFDM users involved in our problem are said to be agents with conflicting interests.

Stated mathematically, $M$ constrained maximization problems have to be simultaneously solved. For each individual problem the optimization variable is the power allocation vector $\mathbf{p}_{i}$ of the transmitter $i \in \mathcal{M}$, and optimal solutions $\mathbf{p}_{i}^{\star}$ depends on the optimal strategic choices $\mathbf{p}_{-i}^{\star}$ made by all others active transmitters:

$$
\begin{aligned}
& \mathbf{p}_{i}^{\star}=\underset{\mathbf{p}_{i}}{\operatorname{argmax}} u_{i}\left(\mathbf{p}_{i}, \mathbf{p}_{-i}^{\star}\right) \quad \forall i \in \mathcal{M} \\
& \text { subject to } \mathbf{p}_{i} \in \mathcal{P}_{i}
\end{aligned}
$$

where $u_{i}\left(\mathbf{p}_{i}, \mathbf{p}_{-i}\right)$ is the objective function of player $i$. The vector $\mathbf{p}_{i}=\left[p_{i}(1), \cdots, p_{i}(N)\right]^{T} \in \mathbb{R}_{+}^{N}$ is the power allocation strategy on the $N$ subcarriers of transmitter $i$, and $\mathbf{p}_{-i}=\left[\mathbf{p}_{1}^{T}, \cdots, \mathbf{p}_{i-1}^{T}, \mathbf{p}_{i+1}^{T}, \cdots, \mathbf{p}_{M}^{T}\right]^{T}$ are the strategies chosen by all the other transmitters. In the following, the concatenated power allocation choices of all players will be called a strategy profile (or a joint strategic choice) and denoted as $\mathbf{p}=\left[\mathbf{p}_{1}^{T}, \cdots, \mathbf{p}_{M}^{T}\right]^{T}$. The set $\mathcal{P}_{i}$ of feasible power allocation strategies of transmitter $i$ is defined as:

$$
\mathcal{P}_{i} \triangleq\left\{\mathbf{p}_{i} \in \mathbb{R}_{+}^{N}: \sum_{n=1}^{N} p_{i}(n) \leq P_{i}^{\max }\right\} .
$$

Section III presents a formulation of the problem as a noncooperative game.

\section{NONCOOPERATIVE SPECTRUM SHARING GAME}

The set of problems (3) can be written as a Nash Equilibrium problem of a noncooperative game $\mathcal{G}$ defined as the following triplet [18]

$$
\mathcal{G} \triangleq\left\{\mathcal{M},\left\{\mathcal{P}_{i}\right\}_{i \in \mathcal{M}},\left\{u_{i}\right\}_{i \in \mathcal{M}}\right\}
$$

where $\mathcal{M}$ is the set of players of the game, $\left\{\mathcal{P}_{i}\right\}_{i \in \mathcal{M}}$ are their strategy spaces, and the objectives $\left\{u_{i}\right\}_{i \in \mathcal{M}}$ are their utility functions. The next step is to formulate utility functions that are compliant with 
the assumptions formulated previously regarding the UA transmission and channel models, and whose noncooperative maximization drives the $M$ OFDM systems towards an efficient spectrum sharing strategy.

A utility function related to the theoretical information rate is chosen here. This choice is reasonable as it suits any type of communication system, without implementation-specific considerations. Because the low propagation speed of acoustic waves coupled with the fast fading part of the channel prohibits the feedback of the instantaneous CSI, this criterion can be related only to some average information rate. Assuming that the duration of $L$ OFDM symbols is much greater than the channel coherence time, a standard figure of merit would be the ergodic capacity [24]

$$
r_{i}\left(\mathbf{p}_{i}, \mathbf{p}_{-i}\right)=\alpha \sum_{n=1}^{N} \mathbb{E}\left\{\log \left(1+\operatorname{SINR}_{i}(n)\right)\right\},
$$

where $\alpha=(N T \Delta f)^{-1}$ and $\operatorname{SINR}_{i}(n)$ is the signal-to-interference plus noise ratio of link $i$ on subcarrier $n:$

$$
\operatorname{SINR}_{i}(n)=\frac{\left|h_{i i}(n)\right|^{2} p_{i}(n)}{\sigma_{w_{i}}^{2}(n)+\sum_{j \neq i}\left|h_{j i}(n)\right|^{2} p_{j}(n)} .
$$

Solving (3) with $r_{i}$ as the utility functions would require that each communication link $i$ has the knowledge of the probability distributions of $h_{j i}(n), \forall j \in \mathcal{M}$ and $\forall n \in\{1, \cdots, N\}$. While realizations of $h_{i i}(n)$ could be estimated at the receiver $i$ using pilot symbols, the interference from transmitters $j \neq i$ cannot be distinguished from noise in the noncooperative setup considered here. To overcome this issue, we adopt a robust approach where the optimization is made considering the most harmful interference. Let $f_{h_{j i}}$ denote the probability density function of $h_{j i}(n)$. The performance metric for the worst case optimization problem can be obtained by minimizing the ergodic capacity according to the cross channels distributions. Thus we define the utility functions $u_{i}$ as

$$
\begin{aligned}
u_{i}\left(\mathbf{p}_{i}, \mathbf{p}_{-i}\right) & =\min _{f_{h_{j i}, i \neq j}: h_{j i} \in \mathcal{L}^{2}} r_{i}\left(\mathbf{p}_{i}, \mathbf{p}_{-i}\right) \\
& =\alpha \sum_{n=1}^{N} \mathbb{E}\left\{\log \left(1+\frac{\left|h_{i i}(n)\right|^{2} p_{i}(n)}{\sigma_{w_{i}}^{2}(n)+\sum_{j \neq i} \mathbb{E}\left\{\left|h_{j i}(n)\right|^{2}\right\} p_{j}(n)}\right)\right\} \\
& \approx \alpha \sum_{n=1}^{N} \log \left(1+\frac{e^{\mathbb{E}\left\{\log \left(\left|h_{i i}(n)\right|^{2}\right)\right\}} p_{i}(n)}{\sigma_{w_{i}}^{2}(n)+\sum_{j \neq i} \mathbb{E}\left\{\left|h_{j i}(n)\right|^{2}\right\} p_{j}(n)}\right),
\end{aligned}
$$

where the last approximation is desirable to derive analytical expressions for the best response strategies of the players (see Section IV). Detailed derivations of (9) and (10) are provided in Appendix A.

No assumption is made regarding the probability distributions of $h_{j i}(n)$. The statistical CSI on each subchannel $n$ will be denoted as

$$
\gamma_{i}(n) \triangleq \frac{e^{\mathbb{E}\left\{\log \left(\left|h_{i i}(n)\right|^{2}\right)\right\}}}{\sigma_{w_{i}}^{2}(n)+\sum_{j \neq i} \mathbb{E}\left\{\left|h_{j i}(n)\right|^{2}\right\} p_{j}(n)},
$$


thus,

$$
u_{i}\left(\mathbf{p}_{i}, \mathbf{p}_{-i}\right)=\alpha \sum_{n=1}^{N} \log \left(1+\gamma_{i}(n) p_{i}(n)\right) .
$$

Note that if one assumes a specific distribution for the channels, (11) may be expressed in closed-form. An example is given in Appendix A where a Rician channel is considered [21], [25], [26].

It must be stressed that $u_{i}$ should not be understood as an achievable rate for UA systems in the sense of [27], [28], but rather as an optimization criterion useful to automatically drive noncooperative UA systems toward a more efficient spectrum sharing strategy than the usual uniform power allocation strategy.

Formulated with $u_{i}$ as the utility functions, solving (3) noncooperatively requires that the communications links only have little and local knowledge about their environment - namely only statistics on their direct subchannel $h_{i i}(n)$ and noise plus aggregate interference power spectral density (PSD). It is important to note that they do not have to distinguish the noise and the interference from other users. Practically, each system would have to estimate its direct channel statistics and the sum of interferences and noise should be treated as one term of additive colored noise. Furthermore, using long-term statistics (e.g., with an integration time of several seconds) can reduce the receiver's feedback activity and cope with issues related to outdated CSI caused by long feedback delays that are typical in UA channels.

\section{ITERATIVE WATER-FILLING SOLUTION}

\section{A. Best-response functions and Nash Equilibrium}

The competitive maximization problem (3) can be solved by finding a Nash Equilibrium (NE) for the game $\mathcal{G}$. In game theory, an NE is a solution concept that is optimal with respect to the selfish behavior of the players. Mathematically, it can be defined as follows [17]:

Definition 1. an NE of the game $\mathcal{G}$ in (5) is a strategy profile $\mathbf{p}^{\star}$ such that $\forall i \in \mathcal{M}$ and $\forall \mathbf{p}_{i} \in \mathcal{P}_{i}$, we have

$$
u_{i}\left(\mathbf{p}_{i}^{\star}, \mathbf{p}_{-i}^{\star}\right) \geq u_{i}\left(\mathbf{p}_{i}, \mathbf{p}_{-i}^{\star}\right)
$$

In the present context, the $M$ UA spectrum users are said to be at an NE when their joint strategic choice $\mathbf{p}^{\star}=\left[\mathbf{p}_{1}^{\star T}, \cdots, \mathbf{p}_{M}^{\star T}\right]^{T}$ of power allocation is such that any link willing to deviate by choosing another $\mathbf{p}_{i} \neq \mathbf{p}_{i}^{\star}$ would experience a loss of information rate. A theorem stated by Debreu, Glicksberg and Fan [29, Theorem 1.2] guarantees the existence of a (pure strategy) equilibrium in any game whose strategic spaces $\mathcal{P}_{i}$ are non-empty compact and convex sub-sets of an Euclidian space and whose utility 
functions $u_{i}\left(\mathbf{p}_{i}, \mathbf{p}_{-i}\right)$ are continuous in $\mathbf{p}$ and quasi-concave in $\mathbf{p}_{i}$. One can verify that the game defined in (5) fulfills these conditions.

Another interpretation of the NE solution can be given by defining the best response of a player to the strategies chosen by the others [18]:

Definition 2. Given a fixed strategic choice $\mathbf{p}_{-i} \in \mathbb{R}_{+}^{N}$ of his opponents, the best responses $B R_{i}\left(\mathbf{p}_{-i}\right)$ of the player $i$ to $\mathbf{p}_{-i}$ is the set of strategies for that player $\mathbf{p}_{i}^{\star}$ defined as

$$
B R_{i}\left(\mathbf{p}_{-i}\right) \triangleq\left\{\mathbf{p}_{i}^{\star} \in \mathbb{R}_{+}^{N}: u_{i}\left(\mathbf{p}_{i}^{\star}, \mathbf{p}_{-i}\right) \geq u_{i}\left(\mathbf{p}_{i}, \mathbf{p}_{-i}\right), \forall \mathbf{p}_{i} \in \mathcal{P}_{i}\right\} .
$$

This means that at an NE, all players $i \in \mathcal{M}$ play their best responses $\mathbf{p}_{i}^{\star} \in B R_{i}\left(\mathbf{p}_{-i}^{\star}\right)$ simultaneously.

The best response function of a player $i$ to a fixed nonnegative $\mathbf{p}_{-i}$ corresponds to the unique solution of (3) and can be found by solving the Karush-Kuhn-Tucker system of equations associated to the dual problem of (3) [30]. This yields the water-filling solution $\forall i \in \mathcal{M}$ and $\forall n \in\{1, \cdots, N\}$

$$
p_{i}^{\star}(n)=\left[\lambda_{i}-\frac{1}{\gamma_{i}(n)}\right]^{+}
$$

where $[x]^{+}$denotes $\max (0, x), \gamma_{i}(n)$ is defined in (11) and $\lambda_{i}$ is the water level chosen by the player $i$ to satisfy its average power constraint (1) with equality.

Thus, an NE of the game $\mathcal{G}$ in (5) is attained when all the communications links adopt the single-user water-filling power allocation strategy against each other, using the statistical CSI (11). In practice, an $\mathrm{NE}$ of $\mathcal{G}$ may emerge as a result of a learning process where all the agents involved in the problem interact by best-responding to each others repeatedly [17], [29]. In our context, this will consist in implementing an iterative water-filling process distributed among the different UA channel users. The sequential water-filling is considered, where the players update their power allocation strategy one after the other according to the solution (15). Note that this particular implementation would not require explicit synchronism between the communication links if we simply assume that the systems have the same update frequency but start their transmission at different times. This choice is primarily motivated by the ease of implementation it offers, but a totally asynchronous update scheme is also possible where the users may have different update periods and have outdated information about the interference PSD. Interested readers are referred to [31], [32] for more details.

Depending on the channel statistics, there can be multiple equilibria and the convergence of iterative best response algorithms may not be guaranteed. Section IV-B is devoted to the analysis of the equilibria of (5) and the convergence of the sequential water-filling algorithm. 


\section{B. Nash Equilibrium uniqueness and convergence of iterative water-filling algorithms}

Sufficient conditions ensuring the uniqueness of the NE and the convergence of iterative water-filling algorithms have been given in [33], [34] in the case of known time-invariant channels. It is possible to follow the same developments to find sufficient conditions on the channel statistics guaranteeing the uniqueness in the game $\mathcal{G}$. Let $\mathbf{Q} \in \mathbb{R}^{M \times M}$ be defined as

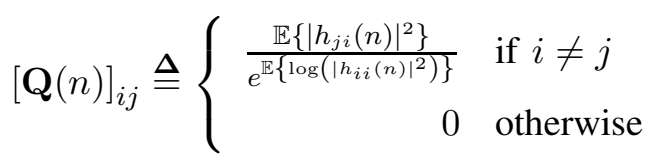

Theorem 1. Game $\mathcal{G}$ admits a unique $N E$ if

$$
\rho(\mathbf{Q}(n))<1, \quad \forall n \in\{1, \cdots, N\}
$$

where $\mathbf{Q}(n)$ is defined in (16) and $\rho(\mathbf{Q}(n))$ is its spectral radius (i.e., the supremum among the absolute values of its eigenvalues).

Proof: See Appendix B.

The convergence of iterative best response algorithms is not guaranteed - not even if the NE is unique - except for some particular types of games [17]. We here focus on sequential water-filling, for which the convergence conditions have been given in [34, Theorem 1] in the case known channel realizations. As for the previous theorem, similar convergence conditions can be derived using the channel statistics. Let $\mathbf{Q}^{\max } \in \mathbb{R}^{M \times M}$ be defined as

$$
\left[\mathbf{Q}^{\max }\right]_{i j} \triangleq\left\{\begin{aligned}
\max _{n} \frac{\mathbb{E}\left\{\left|h_{j i}(n)\right|^{2}\right\}}{e^{\mathbb{E}\left\{\log \left(\left|h_{i i}(n)\right|^{2}\right)\right\}}} & \text { if } i \neq j \\
0 & \text { otherwise }
\end{aligned}\right.
$$

Theorem 2. If the following condition is satisfied

$$
\rho\left(\mathbf{Q}^{\max }\right)<1,
$$

then the sequential water-filling algorithm converges to the unique NE of game $\mathcal{G}$.

Proof: See Appendix C.

These two conditions can be physically interpreted according to the average interference level perceived by each receiver [33]. When the interference is high, (17) holds false. Thus, multiple NE may exist and users will reach one of them by iteratively best-responding to each other (provided that the implemented algorithm converges). The initial power allocation strategy of the players determines which of the NE is finally reached. In this case, as the interferences become stronger, the optimal strategies will tend towards 
the frequency-division multiple access (FDMA)-like power allocations where all the users communicate using orthogonal frequency bands [33], [35]. The sufficient conditions ensuring the uniqueness of the $\mathrm{NE}$ and the convergence of the iterative water-filling can be met when all the users are far enough from each other. However, having (19) not satisfied does not mean that the algorithm cannot drive the players towards a spectrum sharing that is efficient from a practical point of view. As the simulations will show, an iterative water-filling policy can be highly beneficial even if both (17) and (19) hold false.

Up to now, it has been assumed that the channel statistics are known from the players. Section V presents a suitable solution for erroneous knowledge of the statistical CSI in the more realistic situation where it has to be estimated.

\section{Robust GAME}

Solving the game $\mathcal{G}$ with utility functions expressed as in (12) necessitates that each player has a perfect knowledge of the statistical CSI (11). In practice, the receivers would have to estimate the direct channels with pilots symbols. Any estimator produces errors, thus the statistical CSI cannot be perfectly known. However, it is possible to apply the same reasoning as in the case of unknown interference channels by formulating a robust optimization problem against the worst case estimation error. A similar robust rate maximization game under bounded channel uncertainty was also proposed in [36]. Nevertheless a limitation of this work is the assumption that the channel gains $h_{j i}$ are deterministic and known by all the players. This is hardly verified in the UA context. In the following, it is still assumed that the only information that each receiver can retrieve from the multiuser environment is the sum of noise and overall interference PSDs, as well as statistics on its direct channel (between itself and its corresponding transmitter).

We consider an estimator $\hat{\gamma}_{i}(n)$ of $\gamma_{i}(n)$ as a continuous random variable with finite variance such that $\forall n=1, \cdots, N$ we have

$$
\begin{aligned}
\mathbb{E}\left\{\hat{\gamma}_{i}(n)\right\} & =\gamma_{i}(n) \\
\sigma_{\hat{\gamma}_{i}}^{2}(n) & \triangleq \mathbb{E}\left\{\left(\hat{\gamma}_{i}(n)-\gamma_{i}(n)\right)^{2}\right\}
\end{aligned}
$$

The estimator is thus assumed to be unbiased.

The uncertainty on the estimation of the statistical CSI is conveyed by the variance $\sigma_{\hat{\gamma}_{i}}^{2}(n)$. It is assumed that the estimator produces bounded errors. We define the set of statistical CSI producing bounded estimation errors as:

$$
\Gamma_{i} \triangleq\left\{\gamma_{i} \in \mathbb{R}_{+}^{N}:\left|\hat{\gamma}_{i}(n)-\gamma_{i}(n)\right| \leq \epsilon_{i}(n), \forall n=1, \cdots, N\right\},
$$


where $\epsilon_{i}(n)$ is the error bound on the subchannel $n$.

The above assumption is reasonable since the uncertainty on the random variable $\hat{\gamma}_{i}$ can be bounded with high probability by virtue of Bienaymé-Chebychev's inequality. For any $\epsilon_{i}(n) \in \mathbb{R}_{+}$and whatever the probability distribution of $\hat{\gamma}_{i}(n)$ (provided $\sigma_{\hat{\gamma}_{i}}^{2}(n)$ is finite), we have:

$$
\mathbb{P}\left[\left|\hat{\gamma}_{i}(n)-\gamma_{i}(n)\right| \leq \epsilon_{i}(n)\right] \geq 1-\frac{\sigma_{\hat{\gamma}_{i}}^{2}(n)}{\epsilon_{i}^{2}(n)} .
$$

The uncertainty on the values taken by the CSI estimator is taken into account by adopting a robust, maximin power allocation approach. The robust game is thus written as

$$
\tilde{\mathcal{G}} \triangleq\left\{\mathcal{M},\left\{\mathcal{P}_{i}\right\}_{i \in \mathcal{M}},\left\{\tilde{u}_{i}\right\}_{i \in \mathcal{M}}\right\}
$$

where $\left\{\tilde{u}_{i}\right\}_{i \in \mathcal{M}}$ are new utility functions corresponding to the worst case uncertainty, i.e:

$$
\begin{aligned}
& \tilde{u}_{i}\left(\mathbf{p}_{i}, \mathbf{p}_{-i}\right) \triangleq \min _{\boldsymbol{\gamma}_{i} \in \Gamma_{i}} \alpha \sum_{n=1}^{N} \log \left(1+\gamma_{i}(n) p_{i}(n)\right) \\
& =\alpha \sum_{n=1}^{N} \log \left(1+\left[\hat{\gamma}_{i}(n)-\epsilon_{i}(n)\right]^{+} p_{i}(n)\right) .
\end{aligned}
$$

This new game can be solved according to the NE and yields the following robust water-filling solution:

$$
\tilde{p}_{i}^{\star}(n)=\left[\lambda_{i}-\frac{1}{\left[\hat{\gamma}_{i}(n)-\epsilon_{i}(n)\right]^{+}}\right]^{+}, \quad \forall n \in\{1, \cdots, N\} .
$$

The robust game presented here can be viewed as a penalization of the utility functions given in (12) by a term representing some uncertainty about the channels statistics. The effects of such a mecanism will be to make the players more conservative about how they split their power budget on the different subchannels. As the numerical results will show, this behavior can be desirable since it can drive the whole systems towards a more efficient operating point than the NE resulting from the water-filling policy (15).

\section{NUMERICAL RESULTS ON SYNTHETIC CHANNELS}

The benefits of the proposed spectrum sharing method is evaluated by simulation on synthetic UA channels first. UA-OFDM systems accessing the same channel are considered and define the set of players - or users - $\mathcal{M}$ in both games $\mathcal{G}$ and $\tilde{\mathcal{G}}$. Communications take place at the same time and in the same bandwidth $B=6 \mathrm{kHz}$, centered on $f_{c}=12 \mathrm{kHz}$. The bandwidth is divided into $N=256$ subcarriers. The subcarrier spacing is $\Delta f=23.43 \mathrm{~Hz}$, the OFDM block duration is $T=57.7 \mathrm{~ms}$, including a cyclic prefix duration of $T_{g}=15 \mathrm{~ms}$. All the players $i \in \mathcal{M}$ have a power budget $P_{i}^{\max }=N$. In the initial state, they all distribute their power uniformly on the $N$ subcarriers. A reference signal to noise ratio (SNR) 
without interference at a $1 \mathrm{~km}$ transmission distance is fixed to $20 \mathrm{~dB}$ for all users. If the transmission distance varies during the simulations, the SNR varies accordingly to the path loss. The PSD of the noise is modeled as a linear decay of $18 \mathrm{~dB} /$ decade on the frequency scale.

The channels $h_{j i}$ are synthetized using a realistic channel simulator described in [37], [38]. This simulator produces synthetic Rice fading UA channels and proceeds as follows: according to input parameters describing the transmission geometry (namely, ranges and depths), a power-delay profile is obtained via a ray tracing model. Time fluctuations are then generated by entropy maximization of the Doppler spectrum, given as inputs the mean Doppler spread of the fast fading part $\sigma_{D}$ and Rice factor of the main path $K_{\max }$. The Rice factors of the secondary paths decrease exponentially with the delays. For all the simulation scenarios described here, the Doppler spread and Rice factor are set to $\sigma_{D}=1$ $\mathrm{Hz}$ and $K_{\max }=10 \mathrm{~dB}$, respectively. The simulator integrates also physical propagation models for the path losses and frequency-dependent attenuation from absorption (given by Thorp's formula) [4]. Once the impulse responses are generated, the channels coefficients $h_{j i}(n)$ for the subcarriers $n=1, \cdots, N$ are simply obtained via discrete Fourier transform from which the statistics of average channel gains $\mathbb{E}\left\{\left|h_{j i}(n)\right|^{2}\right\}$ and Rice factors $K_{i}(n)$ are extracted to compute the statistical CSI $\gamma_{i}(n)$ given in (38). An example of time-varying frequency responses from the simulator for a two-players game is depicted in Figure 1.
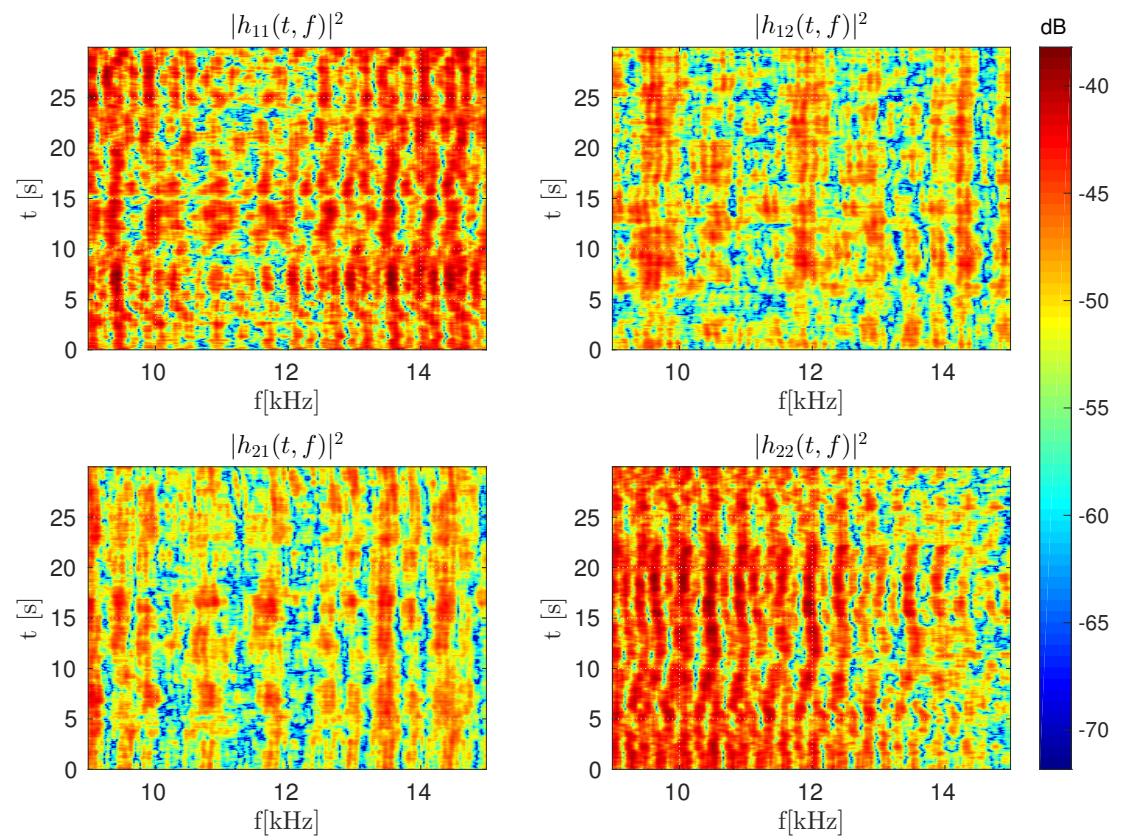

Fig. 1. Time-varying frequency responses of UA channels from the simulator [38]. 
For the game $\mathcal{G}$, perfect knowledge of $\gamma_{i}(n)$ is assumed when the players update their strategy according to the water-filling equation (15). On another hand, it should be estimated when considering the game $\tilde{\mathcal{G}}$. The estimators $\hat{\gamma}_{i}(n)$ are supposed to be unbiased, and their variances $\sigma_{\hat{\gamma}_{i}}^{2}(n)$ can thus be lower bounded by the Cramer-Rao bound on the parameter $\gamma_{i}(n)$. We denote this bound by $I^{-1}\left(\gamma_{i}(n)\right)$, where $I^{-1}$ is the inverse of the Fischer information matrix of $\gamma_{i}(n)$. The estimators $\hat{\gamma}_{i}(n)$ are modeled as Gaussian random variables with mean $\gamma_{i}(n)$ and variances $\sigma_{\hat{\gamma}_{i}}^{2}(n)=R \times I^{-1}\left(\gamma_{i}(n)\right)$, with $R$ a positive constant that allows to generate estimators with different performance, or levels of uncertainty. As the parameter $\gamma_{i}(n)$ is necessarily positive, the Gaussian distribution is truncated to avoid non-positive values of the estimate. The uncertainty bound is then computed as $\epsilon_{i}(n)=k \times \sigma_{\hat{\gamma}_{i}}(n)$, with $k$ a non-negative constant. In order to evaluate systems performance when the estimation errors are not taken into account, we will set $k=0$ and call these games as non-robust games. The water-filling strategies of each player are then computed on the basis of the penalized CSI estimator, as described by equation (26).

Both games are repeated for several iterations. The systems are supposed to start the communications one after the other in the initial state of uniform transmit PSD on the whole bandwidth. Then the game is solved through sequential water-filling: at each iteration $l$, the user $l \bmod M+1$ updates its power allocation strategy according to either (15) or (26), depending on which game is played. The update period of each system is assumed to be equal to the channel observation time, which is here set to $T_{o b s}=30 \mathrm{~s}$. This also corresponds to the period during which the receiver aggregates channel and noise plus interference statistics before feeding it back to its transmitter. Channels statistics are assumed invariant while the game is played.

In the following, systems performance will be expressed according to their utility functions. Though utility functions will be shown in bits per second per hertz (bits/s/Hz) because they are homogeneous to a capacity, they should not be understood as an achievable rate but rather as an optimization metric or as a measure of how good is the actual spectrum sharing for the considered system. The latter interpretation fits well with the meaning of utility functions within the field of game theory [29].

\section{A. Two particular scenarios}

We focus on shallow water scenarios and set the water depth to $50 \mathrm{~m}$. Two-players games modeling different level of interferences are first considered. The depth of each terminal is arbitrarily fixed between 5 and 20 meters and the distance between each transmitter and its intended receiver is $d_{i i}=1 \mathrm{~km}$.

1) High interference setup: A high interference scenario is considered where we set $\forall i, j \neq i \in \mathcal{M}$, $d_{j i} / d_{i i}=1.5$. The path loss from the interfering transmitter and a given receiver is $4 \mathrm{~dB}$ less than from 
the intended transmitter. The channels used to compute the statistical CSI of all users are those of Figure 1. The estimators variances are set to $\sigma_{\hat{\gamma}_{i}}^{2}(n)=R \times I^{-1}\left(\gamma_{i}(n)\right)$ with $R=4$. The uncertainty bound is set to $\epsilon_{i}(n)=k \times \sigma_{\hat{\gamma}_{i}}(n)$ with $k=0$ and $k=3$. The choice $k=3$ guarantees that estimation errors are bounded with probability greater than $88 \%$, whatever their probability distribution (see Eq. (23)). For the Gaussian model chosen here, this probability is equal to $99.7 \%$. The utility functions of the nonrobust and robust games are the sample averages over 100 realizations of the estimators random variables $\hat{\gamma}_{i}$. Figure 2 depicts the utility functions of the two UA OFDM links for both games, evolving with the iterations.

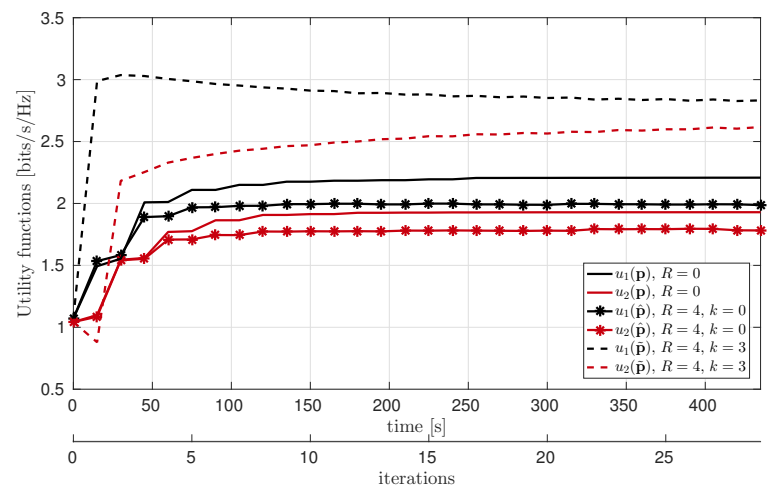

Fig. 2. Utility functions of the players - High interference setup, $d_{j i} / d_{i i}=1.5, d_{i i}=1 \mathrm{~km}$.

The utility values at iteration zero corresponds to the initial state of uniform power allocation. A clear improvement for both users at the end of each game can be seen. Each player have multiplied its initial utility by at least two in the game with perfect knowledge of the statistical CSI $(R=0)$. For both players, more than $90 \%$ of the final utility is reached before 5 iterations, which corresponds to less than 1 min 30 $\mathrm{s}$ considering the $30 \mathrm{~s}$ update period per player. This convergence time could be reduced with a smaller update period but only at the price of increased feedback activity of the receivers and higher estimation variance, since the update period corresponds also to the integration time of the channel statistics.

Note also that in this setup, the sufficient conditions for uniqueness and convergence ((17) and (19)) hold false. In the non-robust game, where estimations errors are not taken into account $(R=4, k=0)$, users reach smaller utilities. However one can see that the robust game $(R=4, k=3)$ yields higher utilities than the game with perfect knowledge of the CSI. Although it seems counter-intuitive, this result can be explained by the conservative behavior of the players in this game. Since their utility functions are penalized by the uncertainty bound $\epsilon_{i}(n)$, they are less inclined to allocate their power on subcarriers 


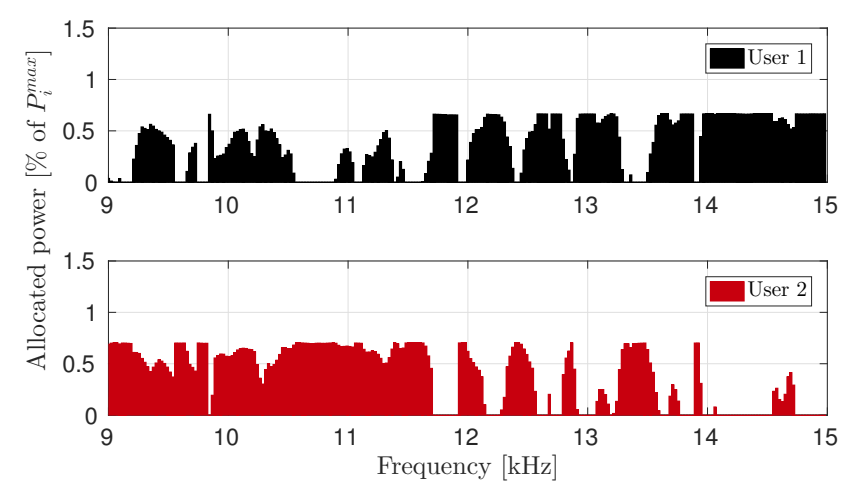

Fig. 3. Last power allocation - Known channel statistics, high interference setup.

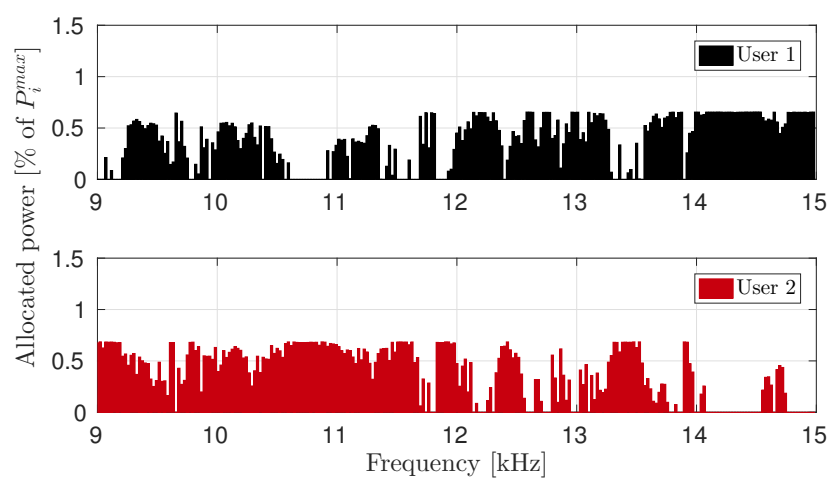

Fig. 4. Last power allocation - nonrobust game for one realization of $\hat{\gamma}_{i}$, high interference setup.

where the channel is not sufficiently good and/or where the interference is too high. Furthermore, each time a user decides to not use a particular subchannel, it lets an extra degree of freedom to the other user for which it should be easier to allocate its power on this particular portion of bandwidth. As a consequence, the robust game can lead to a more efficient spectrum sharing from the point of view of the whole system. This behavior can be clearly seen in Figures 3, 4 and 5 where are shown the power allocations at the end of the game with perfect knowledge, and a realization of the power allocations at the end the nonrobust and robust games, respectively. The power level on each subcarrier is shown in percentage of the average power constraint $P_{i}^{\max }$ of the user.

In the game with perfect knowledge, it can be clearly seen that the allocations strategies are consistent with the frequency channels of Figure 1. For instance, User 2 has more incentive to use the low-frequency subchannels since there is a higher channel gain. Furthermore, as the channel $h_{11}(t, f)$ of the User 1 offers a smaller gain in the band near $11 \mathrm{kHz}$, User 1 has less incentive to use these subcarriers and thus 


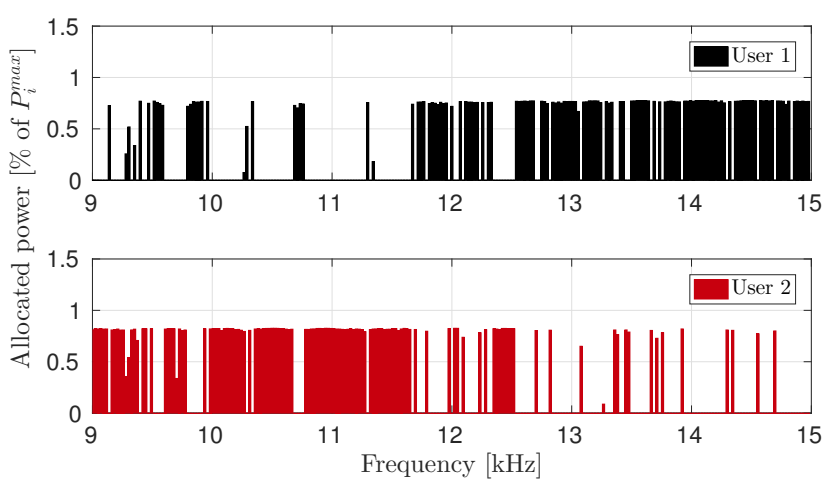

Fig. 5. Last power allocation - Robust game for one realization of $\tilde{\gamma}_{i}$, high interference setup.

generates less interference to User 2. The power allocation of User 1 on subcarriers from 9 to $10 \mathrm{kHz}$ is explained by the interference channel gain $\left|h_{21}(t, f)\right|^{2}$ from transmitter 2 to receiver 1. Roughly speaking, the same spectrum sharing shape can be seen for the nonrobust game in Figure 4. Nevertheless, the power allocations are less correlated with the overall channel state since estimation errors make the players more inclined to simultaneously use the same subchannels. Even if users still interfere on several portions of the spectrum, in the robust game the power allocation strategies tend to be closer to an FDMA-like spectrum sharing. This confirms the conservative behavior of the users in this penalized game.

Interestingly, it has been theoretically proven that a sum-rate optimal ${ }^{3}$ spectrum sharing is FDMA when the crosstalk between the different users is sufficiently high [39]. In the previously cited reference, a locally optimal FDMA power allocation is found by means of a centralized algorithm, assuming the knowledge of all the direct channels. In contrast here, - and recalling what has been shown in [36] in a different context - our present work shows that UA communication links can get close to an FDMA spectrum sharing in an autonomous manner even by assuming only little and possibly erroneous local knowledge about the environment.

2) Low interference setup: In this second setup, a case of lower interferences is considered, where $d_{j i} / d_{i i}=3$. The transmission distance is $d_{i i}=1 \mathrm{~km}$. New channel frequency responses are generated for this scenario. The power received from the interferer is approximately ten times lower than from the transmitter of interest. Robust an non-robust games are run with the same parameters as before. Utility functions are shown in Figure 6.

As the interference power is decreased because of greater distances between the users, these ones are

${ }^{3}$ Or Pareto-optimal, in game-theoretic terms. 


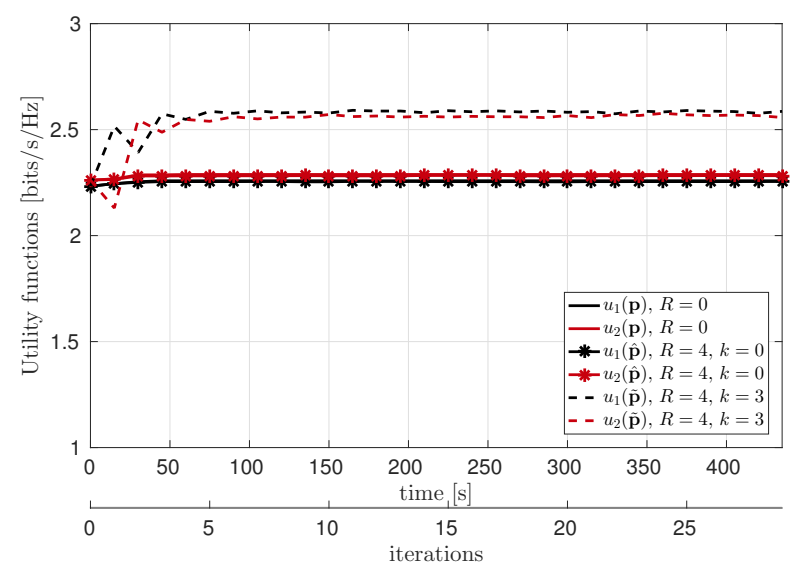

Fig. 6. Utility functions of the players - Low interference, $d_{j i} / d_{i i}=3, d_{i i}=1 \mathrm{~km}$.

less prone to take the multiuser interference into account and share the spectrum using orthogonal bands. Thus, the gain in utility resulting from the water-filling strategy is smaller compared to the initial flat transmit PSD. Nevertheless, the conservative behavior of the players in the robust game is still visible and yields higher values for their utility functions at the final states of the game.

\section{B. General behavior}

The behavior of UA-OFDM users involved in the games $\mathcal{G}$ and $\tilde{\mathcal{G}}$ in more general scenarios is now studied. Focus is put on the performance evaluated in terms of average utility per player as a function of the interference power (or equivalently on the distance separating each receiver from the interfering transmitters), the estimation variance of the CSI, and the number of players in the game. We consider transmissions in an area whose radius varies from $0.5 \mathrm{~km}$ to $8 \mathrm{~km}$. The water depth is still fixed to 50 meters. For each size of the transmission area, 400 games $\mathcal{G}$ and $\tilde{\mathcal{G}}$ were run with terminals immersed at random depths and distances. The minimum distance separating two terminals is fixed to $250 \mathrm{~m}$. The reference SNR is still fixed to $20 \mathrm{~dB}$ at $1 \mathrm{~km}$ and channel parameters $\sigma_{D}, K_{\max }$ remain fixed as specified at the beginning of this section. The resulting utilities are averaged over both the realizations of the transmission geometry and of the random variables $\hat{\gamma}_{i}$.

In order to compare our work with existing, practical spectrum sharing policies, the averaged utilities are also evaluated for

- Single-user water-filling strategies (i.e. considering no interference),

- Naive users adopting the conventional flat power allocation strategy, 


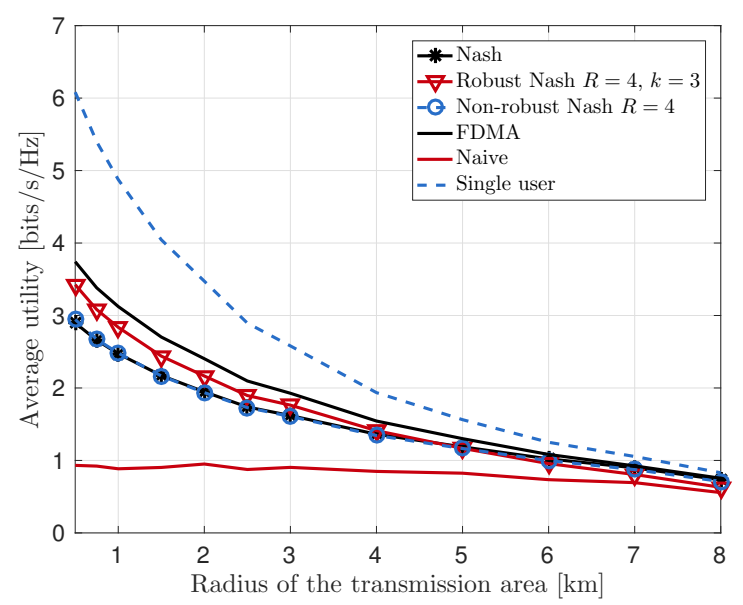

Fig. 7. Average utility per player against the area of the transmission zone. Two players, reference $\mathrm{SNR}=20 \mathrm{~dB}$ at $1 \mathrm{~km}$.

- A FDMA spectrum sharing obtained thanks to a centralized algorithm provided in [39, Algorithm 2], which supposes a perfect statistical knowledge of all the subchannels $h_{i i}(n)$ and of the ambient noise.

1) Influence of the interference power: We first evaluate how the amount of interference affects the behavior of two players by examining how their average utilities evolve with the size of the transmission area. Results are depicted in Figure 7 and show a strong improvement compare to the naive uniform power allocation strategy in small size areas. As the interfering links become far apart, each link has less incentive to share the bandwidth with the others, and the benefits that can be expected from sharing the spectrum decreases with the size of the transmission zone. The power allocation becomes more correlated to the channel frequency-selectivity and the noise than to the behavior of other users. Consequently, the average utility per player tends to the single-user case as the distance between the users tends to infinity. In the robust game, the conservative behavior of all players leads to better operating points which are closer to an optimal FDMA-like spectrum sharing strategy, especially when the interference power is high.

2) Influence of the CSI estimation variance: In order to investigate the influence of the CSI estimators quality on the systems performance, two-player games are run with the same parameters as previously but with different values of $R$ - recalling that $\sigma_{\hat{\gamma}_{i}}^{2}(n)=R \times I^{-1}\left(\gamma_{i}(n)\right)$, with $I^{-1}\left(\gamma_{i}(n)\right)$ the CramerRao Bound on the CSI parameter. Several robust games are compared with the NE solution with perfect knowledge of the channel statistics (dashed lines) and with the FDMA solution proposed in [39, Algorithm 2]. Results are depicted in Figure 8. 


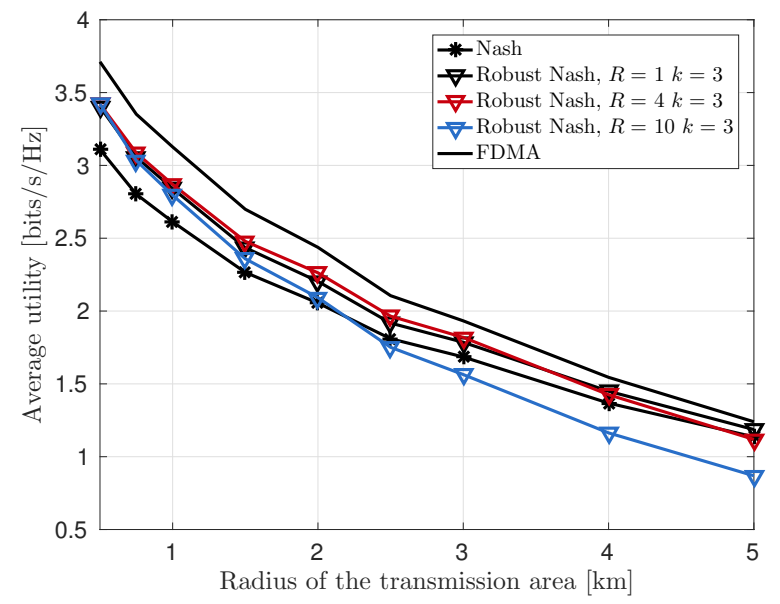

Fig. 8. Influence of the estimators quality on the robust games. Two players, reference SNR $=20 \mathrm{~dB}$ at $1 \mathrm{~km}$.

Interestingly, results show that higher estimators variances $\sigma_{\hat{\gamma}_{i}}^{2}(n)$ (or higher uncertainty about the channel quality) can sometimes yield higher utilities. Though being seemingly counter-intuitive, this can be interpreted as follows: as the estimators variances increases, in some situations the users become more reluctant to allocate power on subcarriers that are not good enough given a fixed uncertainty bound. In other words, since the uncertainty on the channel quality is conveyed by the estimators variances, increasing those variances makes users more conservatives about their power budget. Note also that increasing the interference power implies an increase in estimation variance, which explains why this phenomenon is more often observed in small transmission areas. However, and as the results suggest, there is obviously some threshold above which an higher uncertainty about the channel becomes detrimental.

3) Influence of the number of players: Games are now run with more players and focus is on the average utility per user as a function of their number. The number of user ranges from 2 to 8 . Each terminal is immersed randomly within a circle of $1.5 \mathrm{~km}$ radius, the minimum distance between any transmitter and receiver being constrained to be greater than $250 \mathrm{~m}$. We consider the same three games as before, with estimators $\hat{\gamma}_{i}(n)$ having variances $\sigma_{\hat{\gamma}_{i}}^{2}(n)=4 \times I^{-1}\left(\gamma_{i}(n)\right)$ and uncertainty bounds $\epsilon_{i}(n)=0$ for the non-robust game and $\epsilon_{i}(n)=3 \times \sigma_{\hat{\gamma}_{i}}(n)$ for the robust game. The games are run during 50 iterations from the initial state of uniform transmit PSD on the whole bandwidth for all players. Since the geometry is randomly chosen, the process is repeated 100 times and the utilities of each player at the end of the games are averaged over these 100 realizations.

Results are depicted in Figure 9. Not surprisingly, the average utility per player diminishes as the 


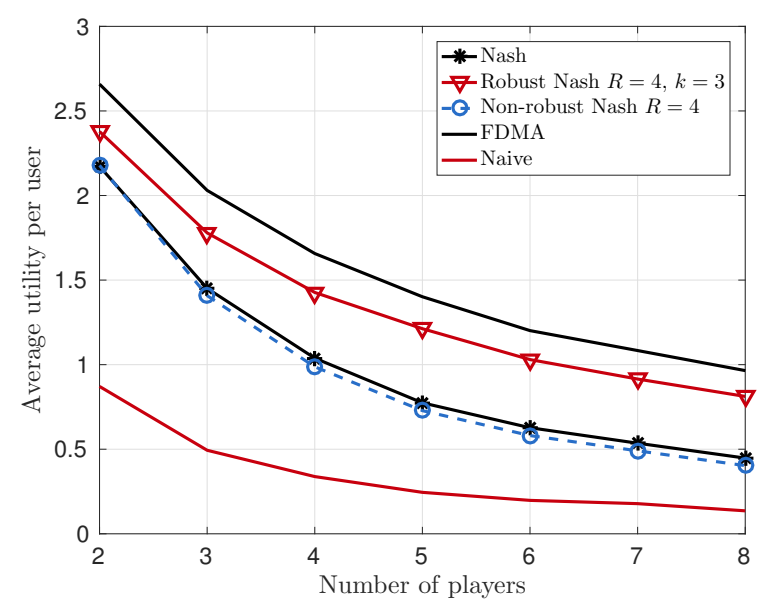

Fig. 9. Average utility per user in function of their number. Each terminal is randomly immersed in a circle of $1.5 \mathrm{~km}$ radius. Reference $\mathrm{SNR}=20 \mathrm{~dB}$ at $1 \mathrm{~km}$.

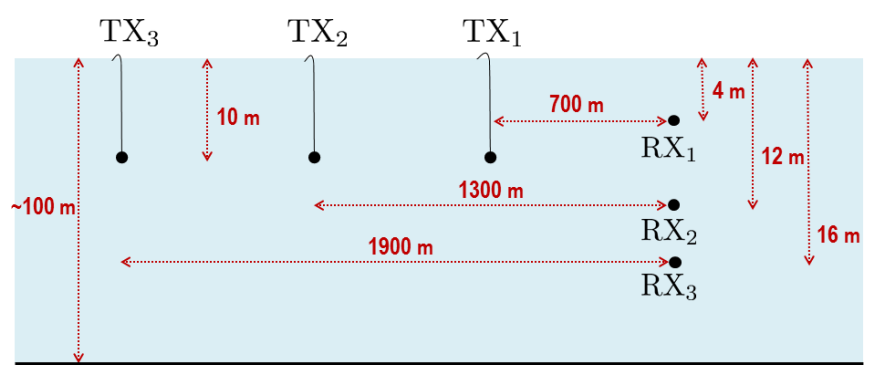

Fig. 10. Configuration of the sea trials.

number of users accessing the same bandwidth in the same geographical area increases. Most importantly, it can be observed that the proposed spectrum sharing methods show a strong improvement in terms of utility compared to the naive strategy and that the robust games still performs better than the perfect knowledge game.

\section{RESULTS ON CHANNELS SOUNDED AT SEA}

\section{A. Experiment setup}

The proposed decentralized power allocation strategy is here illustrated with real channel measurements recorded off the coast of Toulon, France, in July 2015. Three transmitter-receiver pairs were deployed according to the configuration depicted in Figure 10. Three hydrophones RX1, RX2 and RX3 were immersed at depths of $4 \mathrm{~m}, 12 \mathrm{~m}$ and $16 \mathrm{~m}$ respectively, at the same fixed location. The channels from one transmitter to the receivers were sounded at three different locations, corresponding to TX1, TX2 

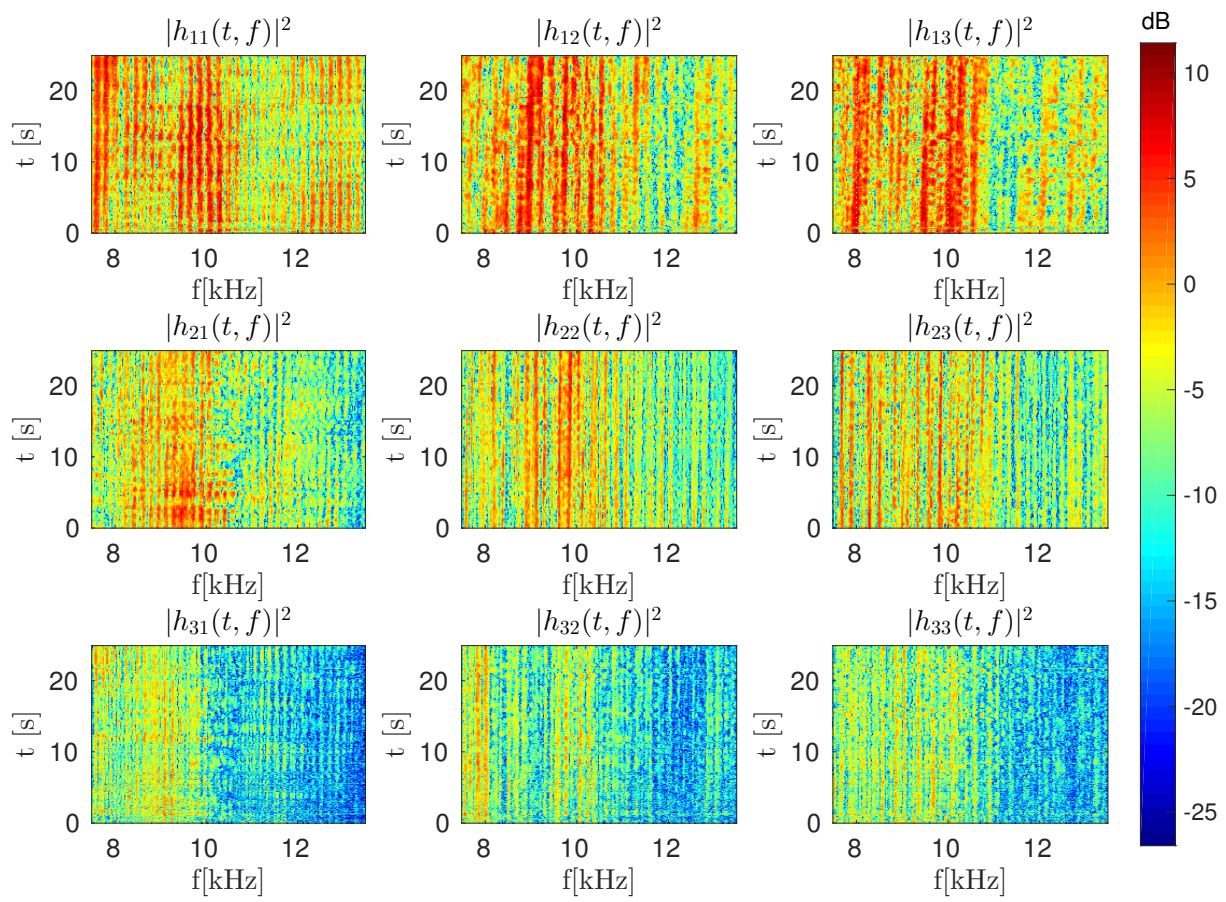

Fig. 11. Measured channels frequency responses.

and TX3. The signal to noise ratios (SNR) for each link were approximately $15 \mathrm{~dB}, 12 \mathrm{~dB}$ and $9 \mathrm{~dB}$ from the nearest to the farthest transmitter.

Measurements of the channel impulse responses were obtained by successive matched filtering to a known probe signal transmitted repeatedly. The probe signal used during the experiments was a msequence of 511 BPSK chips transmitted at a rate of $8.7 \mathrm{kbps}$. Such a sequence can capture arrivals delayed up to $58 \mathrm{~ms}$ and channel estimates can be updated up to 17 times per second. The channel sounding duration was $T_{o b s}=25$ seconds. Measurements were made at a carrier frequency $f_{c}=10.5 \mathrm{kHz}$ and time-varying Doppler shifts were mitigated by the iterative resampling procedure presented in [40], [41]. The processing gain offered by the m-sequence is $27 \mathrm{~dB}$. The measured channels are thus considered as the ground-truth from the point of view of the multiuser system simulated next. Consequently, their Rice factors and averaged frequency responses are said to be the perfectly estimated statistics in the following. Thus, the game $\mathcal{G}$ will be run with players based on this knowledge when they implement sequentially the water-filling strategy (15). The time-varying frequency responses in a $6 \mathrm{kHz}$ bandwidth centered on $f_{c}$ are shown in Figure 11. The Rice factors $K_{i}(n)$ for the direct frequency subchannels are between 1.4 $\mathrm{dB}$ and $6 \mathrm{~dB}$ in average depending on the link, and with higher Rice factors for subchannels with high 


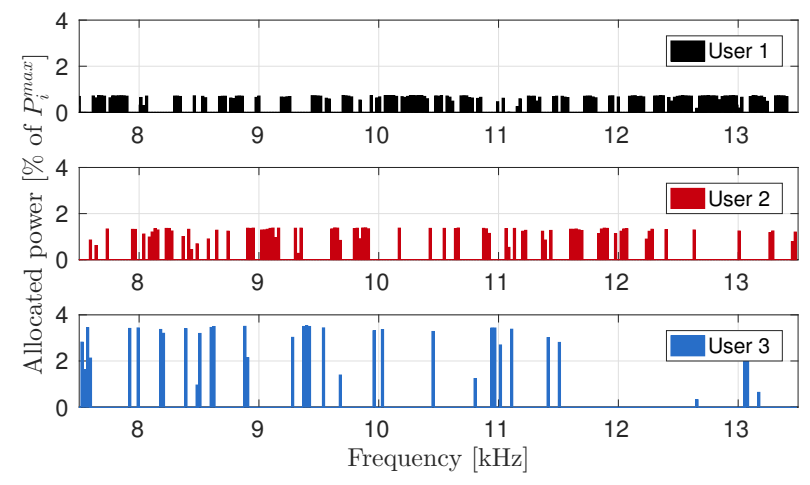

Fig. 12. Power allocations at the end of the games - Real channels, robust game.

gains.

\section{B. Simulation of the spectrum sharing game}

The behavior of three UA OFDM links implementing the distributed water-filling solutions (15) and (26) is simulated next, on the basis of the channels sounded at-sea. The three users share the same bandwidth $B=6 \mathrm{kHz}$ centered on $f_{c}$ and divided into $N=256$ subcarriers. The subcarriers spacing, block duration and cyclic prefix duration are the same as for the simulations on synthetic channels. Each user has a power constraint $P_{i}^{\max }=N$. The initial states for both games $\mathcal{G}$ and $\tilde{\mathcal{G}}$ are the uniform power allocation strategy for the three users. Games are then run during 30 iterations, on the same basis as the simulations on synthetic channels. The period at which each user updates its strategy is equal to the channel observation time of $25 \mathrm{~s}$. The users play one after the other and only one user plays per iteration. Thus, the update period corresponds to three consecutive iterations. Each user implements its water-filling based on the CSI given in (38). ${ }^{4}$ For the nonrobust and robust games, the estimators variances are set to $\sigma_{\hat{\gamma}_{i}}^{2}(n)=R \times I^{-1}\left(\gamma_{i}(n)\right)$ with $R=4$ and the uncertainty bounds are $\epsilon_{i}(n)=k \times \sigma_{\hat{\gamma}_{i}}(n)$ with $k=2$. The value chosen for the latter parameter guarantees that the estimators $\hat{\gamma}_{i}(n)$ produce errors below the uncertainty bound with probability equal to $95.4 \%$

The last power allocations of the robust game are depicted in Figure 12, which are consistent with the frequency responses of Figure 11. User 1 takes advantage of the transmission geometry, it has less incentive to share the bandwidth with its competitors and thus occupies more subchannels. Therefore,

\footnotetext{
${ }^{4}$ Tests have been conducted and have shown that no significant differences in the results can be seen whether Rice fading is assumed or not in this experiment.
} 


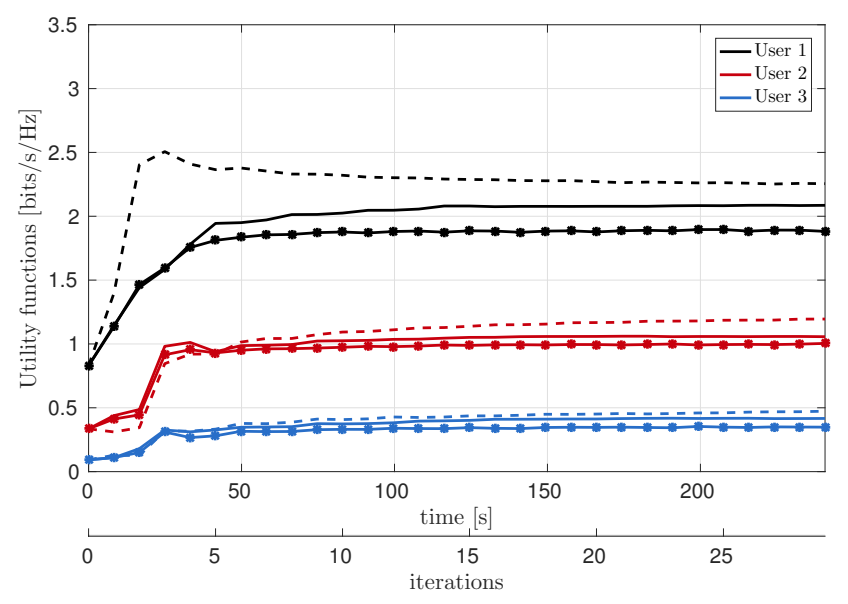

Fig. 13. Utility functions of the players - Real channels. Plain line : perfect knowledge of the statistical CSI, stars : nonrobust game $(R=4, k=0)$, dashed : robust game $(R=4, k=2)$.

Users 2 and 3 have to share the subcarriers where User 1 has allocated less or no power. Having its best channel gain between $7.5 \mathrm{kHz}$ and $10.5 \mathrm{kHz}$, its not surprising that User 3 has more incentive to allocate its power on these subcarriers, avoiding the ones where User 1 is already present. The power allocation of User 2 is more difficult to interpret. This player has the disadvantage to experience better channel gains on subcarriers where both $\left|h_{11}(t, f)\right|^{2}$ and $\left|h_{12}(t, f)\right|^{2}$ are high: these subchannels are both those User 1 is inclined to use and through which User 2 experiences most interference from that player. On another hand, Users 2 and 3 interfere the most to User 1 on subcarriers ranging from $8 \mathrm{kHz}$ to $10.5 \mathrm{kHz}$. Consequently they have some opportunity to push User 1 out of some subcarriers on this band. This is particularly true in the robust game, thanks to the conservative behavior of User 1.

Utilities achieved at each iterations for both games are shown in Figure 13. At the end of the game, all users have multiplied their utilities by at least two in the game with perfect knowledge of the statistical CSI. As expected, performance are diminished in the nonrobust game. Estimations errors are less harmful for players 2 and 3 compared to player 1, their situations regarding the transmission geometry being the preponderant factor on their performance. In this particular setup, all players in the robust game have increased their utilities, even above those in the perfect knowledge case. This confirms with experimental data that the robust game proposed is able to reach operating points that are Pareto superior to the Nash equilibrium without information exchange between players, cooperation, or centralized control. 


\section{PRACTICAL ASPECTS}

Some practical aspects related to convergence time of iterative water-filling algorithm to an equilibrium, CSI estimation and channel stationarity assumptions must be stressed. It should be mentioned that these three parameters are linked together by the choice of the period of time $T_{o b s}$ on which the channel statistics are integrated, which corresponds also to the update period of the power allocation strategies of each player. In a practical setup, each player could have its own update period which results in an asynchronous water-filling algorithm rather than the sequential update we presented so far. However, this does not change the following comments. The channel stationarity time should be greater than the convergence time of the iterative water-filling algorithm. It is then expected that the iterative nature of the procedure is able to track the larger scale fading. For the sequential algorithm, convergence time is mainly dependent on the update period $T_{o b s}$. Provided that the channel statistics are indeed stationary, increasing this period should reduce the CSI estimation variance and thus produce a spectrum sharing that fits better to the global channel state. However, this would be done at the price of a longer convergence time. In addition, the period $T_{o b s}$ should be chosen large in comparison to the feedback link delay which depends on the distance between a receiver and its transmitter. Note that if the channel statistics are not stationary during the whole period $T_{o b s}$, this would result in an estimation error against which the robust game can provide a solution. Finally, the more users there are sharing underwater channel in the same area, the more difficult it will be to reach an equilibrium (recall also that the conditions (17) and (19) on the uniqueness of an NE and the convergence of iterative algorithms would hardly be met).

\section{CONCLUSION}

An efficient spectrum sharing method distributed between interfering UA OFDM systems has been proposed. Tools from game theory allowed us to devise a noncooperative solution, which only necessitates that each communication link has a local and statistical knowledge about its environment. The proposed method copes with realistic constraints of the UA context - namely, the impossibility for systems in different networks to exchange information about their channel states, the double selectivity and the random nature of most UA channels, as well as the low propagation speed of UA waves. Erroneous knowledge of the statistical CSI has also been taken into account by a robust formulation of the initial game. Utility functions are minimized on the set of CSI for which estimators lie within an uncertainty bound that can be parameterized. Simulation results on synthetic and real UA channels have shown the effectiveness of the proposed spectrum sharing strategy. In medium and high interference setups, utility functions of the players can be multiplied by at least a factor of two compared to the naive uniform 
transmit PSD. Furthermore, in some situations, it is possible to get close to the FDMA strategy while requiring much less information to be known by the players. Though real and synthetic shallow water channels have been considered for simulations, the same spectrum management strategy could also be applied in deep waters. Many extensions of this work are possible. First, it should be noticed that the Nash Equilibrium solution proposed here is unfair in the sense that the achieved spectrum sharing could be good for some users and not for the others. Simulations on real channels have shown that a player can take advantage of its initial situation without caring for the other systems evolving in the same area. This calls for the study of mechanisms able to improve the equilibrium efficiency or even create implicit cooperation between players. Games with pricing could be a path of investigation toward decentralized and noncooperative method to reach operating points that are Pareto superior to the NE. The quantity of information that receivers have to feedback can also be a problem for practical systems. A complementary but similar game has been studied in another work [42], where UA-OFDM communication links are aimed at minimizing their transmission power under information rate constraint. A natural extension of both [42] and this paper are satisfaction games [43], [44] where players do not aim at maximizing their utility but only at finding a strategy that guarantees a minimum satisfaction level. This type of game could reduce the feedback information to only one bit. Further extensions of this works could also consider interactions between heterogeneous systems, e.g sonars and communications systems. To conclude, future works on the topic should also evaluate the benefits of our method for practical system implementations. Measuring the possible BER improvements provided by our approach during at-sea trials by considering practical coding and modulation schemes could be a relevant example.

\section{Acknowledgements}

The authors are grateful to DGA-TN for providing the experimental data used in this paper. This work has been partially funded by Région Bretagne.

\section{APPENDIX A}

$$
\text { DERIVATION OF UTILITY FUNCTIONS } u_{i}\left(\mathbf{p}_{i}, \mathbf{p}_{-i}\right)
$$

\section{A. Minimization Against the Worst Case Cross-Channels Distributions}

The ergodic capacity of the player $i$ is given by

$$
r_{i}\left(\mathbf{p}_{i}, \mathbf{p}_{-i}\right) \propto \sum_{n=1}^{N} \mathbb{E}\left\{\log \left(1+\operatorname{SINR}_{i}(n)\right)\right\}
$$


with $\operatorname{SINR}_{i}(n)$ defined by

$$
\operatorname{SINR}_{i}(n)=\frac{\left|h_{i i}(n)\right|^{2} p_{i}(n)}{\sigma_{i}^{2}(n)+\sum_{j \neq i}\left|h_{j i}(n)\right|^{2} p_{j}(n)}
$$

and where the expectation in (27) is evaluated according to the joint probability distribution of the $h_{j i}, i, j \in \mathcal{M}$.

The proposed utility function is

$$
u_{i}\left(\mathbf{p}_{i}, \mathbf{p}_{-i}\right)=\min _{f_{h_{j i}, i \neq j}: h_{j i} \in \mathcal{L}^{2}} r_{i}\left(\mathbf{p}_{i}, \mathbf{p}_{-i}\right)
$$

To solve this probem, the minimum ergodic capacities of all subchannels $n \in\{1, \cdots, n\}$ can be equivalently found.

$$
\begin{aligned}
\mathbb{E}\left\{\log \left(1+\operatorname{SINR}_{i}(n)\right)\right\} & \stackrel{(a)}{=} \mathbb{E}_{h_{i i}}\left\{\mathbb{E}_{h_{j \neq i, i}}\left[\log \left(1+\frac{\left|h_{i i}(n)\right|^{2} p_{i}(n)}{\sigma_{i}^{2}(n)+\sum_{j \neq i}\left|h_{j i}(n)\right|^{2} p_{j}(n)}\right) \mid h_{i i}\right]\right\} \\
& \stackrel{(b)}{\geq} \mathbb{E}_{h_{i i}}\left\{\log \left(1+\frac{\left|h_{i i}(n)\right|^{2} p_{i}(n)}{\sigma_{i}^{2}(n)+\sum_{j \neq i} \mathbb{E}_{h_{j i}}\left\{\left|h_{j i}(n)\right|^{2}\right\} p_{j}(n)}\right)\right\}
\end{aligned}
$$

Using (28) and conditional expectations, we can write equation (30)-(a). Inequality (30)-(b) then follows from Jensen's inequality, since the function under the conditional expectation is convex in $h_{j i}(n)$. The solution of problem (29) is given by the right-hand side of inequality (30)-(b), which is an equality for gaussian interference (a commonly used assumption in practical systems). This proves (9).

The game could be defined with (9) as utility functions. However, the water-filling solution would not have a closed-form expression as in (15) and convergence and NE uniqueness conditions cannot be found easily. This justifies the need to obtain a utility function expressed as the right member of (10). We first rewrite the utility function as

$$
\begin{aligned}
u_{i}\left(\mathbf{p}_{i}, \mathbf{p}_{-i}\right) & =\sum_{n=1}^{N} \mathbb{E}_{h_{i i}}\left\{\log \left(1+\frac{\left|h_{i i}(n)\right|^{2} p_{i}(n)}{\zeta_{i}(n)}\right)\right\} \\
& =\sum_{n=1}^{N} \mathbb{E}_{h_{i i}}\left\{\log \left(1+\frac{e^{\log \left|h_{i i}(n)\right|^{2}} p_{i}(n)}{\zeta_{i}(n)}\right)\right\}
\end{aligned}
$$

where,

$$
\zeta_{i}(n)=\sigma_{i}^{2}(n)+\sum_{j \neq i} \mathbb{E}\left\{\left|h_{j i}(n)\right|^{2}\right\} p_{j}(n) .
$$

Noticing that $u_{i}\left(\mathbf{p}_{i}, \mathbf{p}_{-i}\right)$ is then convex in $\log \left|h_{i i}(n)\right|^{2}$ yields the following inequality

$$
u_{i}\left(\mathbf{p}_{i}, \mathbf{p}_{-i}\right) \geq \sum_{n=1}^{N} \log \left(1+\frac{e^{\mathbb{E}\left\{\log \left|h_{i i}(n)\right|^{2}\right\}} p_{i}(n)}{\zeta_{i}(n)}\right),
$$


which ends the proof. It can be shown numerically that, with the real data used in Sec. VII or under under Rice fading assumptions, (32) is a tight lower bound of (31).

\section{B. Approximation Under the Rician Fading Channel Assumption}

If one makes the choice to model the UA channel fading process as Rician, we have that $h_{i i}(n) \sim$ $\mathcal{C N}\left(\mu_{i i}(n), \sigma_{i i}^{2}(n)\right) \forall n \in\{1, \cdots, N\}$ and thus their squared modulus follow a non-central chi-squared law. Inequality (32) can be rewritten using the expected logarithm of non-central chi-squared variable formula, which can be found in [45, Equation (209)]. Let us recall this formula and adapt it to our needs and notations in the following theorem:

Theorem 3. Let $H \sim \mathcal{C N}(0,1), \mu \in \mathbb{C}$ and $\sigma \in \mathbb{R}_{+} \backslash\{0\}$. Then, we have

$$
\begin{aligned}
\mathbb{E}\left\{\log |\mu+\sigma H|^{2}\right\}= & \log \sigma^{2}+\log \frac{|\mu|^{2}}{\sigma^{2}} \\
& -\operatorname{Ei}\left(-\frac{|\mu|^{2}}{\sigma^{2}}\right) .
\end{aligned}
$$

with Ei $(-x)$ the exponential integral function defined, for $x>0$, as

$$
\operatorname{Ei}(-x) \triangleq-\int_{x}^{+\infty} \frac{e^{-t}}{t} d t
$$

It is possible to rewrite $h_{i i}(n)=\mu_{i i}(n)+\sigma_{i i}(n) H$ with $H \sim \mathcal{C N}(0,1)$. The Rice factor $K_{i}(n)$ and channel gain of $h_{i i}(n)$ are defined as

$$
\begin{aligned}
& K_{i}(n) \triangleq \frac{\left|\mu_{i i}(n)\right|^{2}}{\sigma_{i i}^{2}(n)} \\
& \mathbb{E}\left\{\left|h_{i i}(n)\right|^{2}\right\}=\left|\mu_{i i}(n)\right|^{2}+\sigma_{i i}^{2}(n) .
\end{aligned}
$$

Applying Theorem 3 to the expected logarithm in inequality (31) with the two definitions above yields

$$
\begin{aligned}
\mathbb{E}\left\{\log \left|h_{i i}(n)\right|^{2}\right\}=\log \sigma_{i i}^{2}(n)+\log K_{i}(n)-\operatorname{Ei}\left(-K_{i}(n)\right) \\
=\log \left(\frac{K_{i}(n)}{K_{i}(n)+1} \mathbb{E}\left\{\left|h_{i i}(n)\right|^{2}\right\}\right) \\
-\operatorname{Ei}\left(-K_{i}(n)\right) .
\end{aligned}
$$

Equation (11) then becomes

$$
\gamma_{i}(n)=\frac{g_{i}(n) \mathbb{E}\left\{\left|h_{i i}(n)\right|^{2}\right\}}{\sigma_{w_{i}}^{2}(n)+\sum_{j \neq i} \mathbb{E}\left\{\left|h_{j i}(n)\right|^{2}\right\} p_{j}(n)},
$$


where

$$
g_{i}(n)=\frac{K_{i}(n)}{K_{i}(n)+1} e^{-\operatorname{Ei}\left(-K_{i}(n)\right)} .
$$

Note that no consensus exists regarding the statistical modeling of UA communication channels. The interested reader is referred to [21], [25], [26], [46], [47], which - among other works - can give an idea of the variety of existing statistical channel models for UA communications. Under the Rician fading assumption, the expression (38) only depends on channel parameters - the Rice factor and channel gain - for which efficient estimation methods exist (see for instance [48] for a practical Rice factor estimation method).

\section{APPENDIX B}

\section{Proof of THEOREM 1: UniQUENESS OF THE NASH EQUILIBRUM}

In general, only sufficient conditions can be given for guaranteeing NE uniqueness in games whose utility functions are concave and strategy spaces are closed, convex and bounded. These conditions can be obtained by prooving that utility functions are diagonally strictly concave, which imply NE uniquess. This notion of diagonal strict concavity has been first introduced in [49]. We recall this notion and adapt it to our needs in the following theorem:

Theorem 4. Let $\mathcal{G}=\left\{\mathcal{M}, \mathcal{P}=\mathcal{P}_{1} \times \cdots \times \mathcal{P}_{M},\left(u_{i}\right)_{i=1}^{M}\right\}$ be a game with convex, closed and bounded strategic spaces $\mathcal{P}_{i}$ and utility functions being continuous in $\mathbf{p} \in \mathcal{P}$ and concave in $\mathbf{p}_{i} \in \mathcal{P}_{i}, \forall i \in \mathcal{M}$. Any vector $\mathbf{p} \in \mathcal{P}$ can be decomposed as $\mathbf{p}=\left[\mathbf{p}_{1}^{T}, \cdots, \mathbf{p}_{M}^{T}\right]^{T}$. If $\forall \mathbf{p}, \mathbf{p}^{\prime} \in \mathcal{P}, \mathbf{p} \neq \mathbf{p}^{\prime}, \exists i \in \mathcal{M}$ such that the following holds

$$
\left(\mathbf{p}_{i}^{\prime}-\mathbf{p}_{i}\right)^{T} \nabla_{\mathbf{p}_{i}} u_{i}(\mathbf{p})+\left(\mathbf{p}_{i}-\mathbf{p}_{i}^{\prime}\right)^{T} \nabla_{\mathbf{p}_{i}} u_{i}\left(\mathbf{p}^{\prime}\right)>0
$$

then the Nash Equilibrum of game $\mathcal{G}$ is unique.

Proof: See [49, Theorem 2].

Then, the main idea is to suppose two different NEs $\mathbf{p}, \mathbf{p}^{\prime} \in \mathcal{P}=\mathcal{P}_{1} \times \cdots \times \mathcal{P}_{M}$ and to find what are the conditions on the channel statistics that satisfy Theorem 4.

First, $u_{i}\left(\mathbf{p}_{i}, \mathbf{p}_{-i}\right)$ is rewritten as

$$
u_{i}\left(\mathbf{p}_{i}, \mathbf{p}_{-i}\right) \propto \sum_{n=1}^{N} \log \left(1+\frac{H_{i i}(n) p_{i}(n)}{\sigma_{i}^{2}(n)+\sum_{j \neq i} H_{j i}(n) p_{j}(n)}\right)
$$


where

$$
H_{j i}(n) \triangleq \begin{cases}e^{\mathbb{E}\left\{\log \left|h_{i i}(n)\right|^{2}\right\}} & \text { if } i=j \\ \mathbb{E}\left\{\left|h_{j i}(n)\right|^{2}\right\} & \text { otherwise }\end{cases}
$$

We have $\forall n \in\{1, \cdots, N\}$,

$$
\left[\nabla_{\mathbf{p}_{i}} u_{i}(\mathbf{p})\right]_{n}=\frac{H_{i i}(n)}{\sigma_{i}^{2}(n)+\sum_{j=1}^{M} H_{j i}(n) p_{j}(n)} .
$$

Let $\mathbf{p}, \mathbf{p}^{\prime} \in \mathcal{P}=\mathcal{P}_{1} \times \cdots \times \mathcal{P}_{M}$ and suppose $\mathbf{p} \neq \mathbf{p}^{\prime}$. Using the above definitions in condition (40) yields that $\mathbf{p}$ and $\mathbf{p}^{\prime}$ cannot be both NE if $\exists i \in \mathcal{M}$ such that

$$
\sum_{n=1}^{N}\left(p_{i}^{\prime}(n)-p_{i}(n)\right) \frac{H_{i i}(n)}{\xi_{i}(n)} \times \sum_{j=1}^{M} H_{j i}(n)\left(p_{j}^{\prime}(n)-p_{j}(n)\right)>0
$$

where

$$
\xi_{i}(n)=\left(\sigma_{i}^{2}(n)+\sum_{j=1}^{M} H_{j i}(n) p_{j}(n)\right) \times\left(\sigma_{i}^{2}(n)+\sum_{j=1}^{M} H_{j i}(n) p_{j}^{\prime}(n)\right)>0 .
$$

It can be shown that [33, Appendix B] a sufficient condition to have (44) true is $\exists i \in \mathcal{M}, \forall n \in$ $\{1, \cdots, N\}$

$$
\left|p_{i}^{\prime}(n)-p_{i}(n)\right|+\operatorname{sign}\left(p_{i}^{\prime}(n)-p_{i}(n)\right) \times \sum_{j \neq i} G_{j i}(n)\left(p_{j}^{\prime}(n)-p_{j}(n)\right)>0
$$

with $G_{j i}(n)$ defined for $j \neq i$ as

$$
G_{j i}(n) \triangleq \frac{H_{j i}(n)}{H_{i i}(n)}, \quad \forall n \in\{1, \cdots, N\}
$$

Starting from this point, the same steps as in [33, Appendix B] can be applied and yield the condition of Theorem 1 when translated in terms of the channel statistics.

\section{APPENDIX C}

\section{PROOF OF THEOREM 2: CONVERGENCE OF ITERATIVE WATER-FILLING}

The water-filling operator can be seen from the point of view of the whole system as a mapping from $\mathcal{P}=\mathcal{P}_{1} \times \cdots \times \mathcal{P}_{M}$ to itself. Let $\mathbf{p}=\left[\mathbf{p}_{1}^{T}, \cdots, \mathbf{p}_{M}^{T}\right] \in \mathcal{P}$ be the concatenation of all the power allocation vectors $\mathbf{p}_{i} \in \mathcal{P}_{i}$. The water-filling mapping can be defined as

$$
W F(\mathbf{p})=\left(W F_{i}\left(\mathbf{p}_{-i}\right)\right)_{i=1}^{M}
$$


where $\forall n \in\{1, \cdots, N\}$

$$
\left[W F_{i}\left(\mathbf{p}_{-i}\right)\right]_{n}=\left[\lambda_{i}-\frac{\sigma_{i}^{2}(n)+\sum_{j \neq i} \mathbb{E}\left\{\left|h_{j i}(n)\right|^{2} p_{j}(n)\right\}}{e^{\mathbb{E}\left\{\log \left(\left|h_{i i}(n)\right|^{2}\right)\right\}}}\right]^{+} .
$$

an NE $\mathbf{p}^{\star}$ of the game $\mathcal{G}$ is by definition a fixed point of the best response mapping defined by (48) [18, Proposition 3.1], i.e.

$$
\mathbf{p}^{\star}=W F\left(\mathbf{p}^{\star}\right)
$$

The key argument to prove convergence of iterative algorithms based on the mapping (48) to an NE is to show that this mapping is a block-contraction (see [34, Appendices B and C]). We recall this notion in the following definitions [50]:

Definition 3 (Block-maximum norm). Let $\mathcal{X}=\mathcal{X}_{1} \times \cdots \times \mathcal{X}_{M} \subseteq \mathbb{R}^{n}$ where each $\mathcal{X}_{i}$ is a nonempty Euclidean subspace of $\mathbb{R}^{n_{i}}$ endowed with the Euclidean norm denoted by $\|\cdot\|_{2}$, and where $n=n_{1}+$ $\cdots+n_{M}$. Any vector $\mathbf{x} \in \mathcal{X}$ can be decomposed as $\mathbf{x}=\left[\mathbf{x}_{1}^{T}, \cdots, \mathbf{x}_{M}^{T}\right]^{T}$. The block-maximum norm on $\mathbb{R}^{n}$ is defined as

$$
\|\mathbf{x}\|_{\text {block }}=\max _{i}\left\|\mathbf{x}_{i}\right\|_{2}
$$

Definition 4 (Block-contraction mapping). Let $T: \mathcal{X} \rightarrow \mathcal{X}$ where $\mathcal{X}$ is defined as in Definition 3. Any vector $\mathbf{x} \in \mathcal{X}$ can be decomposed as $\mathbf{x}=\left[\mathbf{x}_{1}^{T}, \cdots, \mathbf{x}_{M}^{T}\right]^{T}$ and $\forall \mathbf{x} \in \mathcal{X}$ we have $T(\mathbf{x})=$ $\left[T_{1}(\mathbf{x}), \cdots, T_{M}(\mathbf{x})\right]$ where each $T_{i}: \mathcal{X} \rightarrow \mathcal{X}_{i}$ is the $i^{\text {th }}$ block-component of $T$. ( $T$ is called a blockcontraction mapping of modulus $\beta$ if it verifies $\forall \mathbf{x}, \mathbf{y} \in \mathcal{X}$

$$
\|T(\mathbf{x})-T(\mathbf{y})\|_{\text {block }} \leq \beta\|\mathbf{x}-\mathbf{y}\|_{\text {block }}
$$

with $\beta \in[0,1)$. Such a mapping has a unique fixed-point $\mathbf{x}^{\star}=T\left(\mathbf{x}^{\star}\right)$.

Suppose now a sequential algorithm to find a fixed point of such mapping $T$, which updates a vector $\mathbf{x}$ on a block-wise basis, i.e. at each iteration $t \in \mathbb{N}$ we have $\forall i \in\{1, \cdots, M\}$

$$
\mathbf{x}_{i}(t+1)=\left\{\begin{aligned}
T_{i}(\mathbf{x}(t)) & \text { if }(t+1) \bmod M=i \\
\mathbf{x}_{i}(t) & \text { otherwise. }
\end{aligned}\right.
$$

Such an algorithm is called the Gauss-Seidel algorithm based on mapping $T$ [50]. We have now the following convergence theorem:

Theorem 5 (Convergence of Gauss-Seidel Block Iterations [50]). If $T: \mathcal{X} \rightarrow \mathcal{X}$ is a block-contraction mapping, the sequence computed by the Gauss-Seidel algorithm (53) based on mapping T converges geometrically to the unique fixed point of $T$ from any initial point. 
The sequential iterative water-filling described in Section IV can be identified to a Gauss Seidel implementation of the water-filling mapping defined in (48). Using the interpretation of the water-filling operator as an Euclidean projector on a convex set [34] we can rewrite (49) as

$$
W F_{i}\left(\mathbf{p}_{-i}\right)=\left[-\boldsymbol{\sigma}_{i}-\sum_{j \neq i} \mathbf{H}_{j i} \mathbf{p}_{j}\right]_{\mathcal{P}_{i}}
$$

where

$$
\begin{aligned}
& \boldsymbol{\sigma}_{i} \triangleq\left[\frac{\sigma_{i}^{2}(1)}{e^{\mathbb{E}\left\{\log \left(\left|h_{i i}(1)\right|^{2}\right)\right\}}}, \cdots, \frac{\sigma_{i}^{2}(N)}{e^{\mathbb{E}\left\{\log \left(\left|h_{i i}(N)\right|^{2}\right)\right\}}}\right]^{T}, \\
& \mathbf{H}_{j i} \triangleq \operatorname{diag}\left(\left[\frac{\mathbb{E}\left\{\left|h_{j i}(1)\right|^{2}\right\}}{e^{\mathbb{E}\left\{\log \left(\left|h_{i i}(1)\right|^{2}\right)\right\}}}, \cdots, \frac{\mathbb{E}\left\{\left|h_{j i}(N)\right|^{2}\right\}}{e^{\mathbb{E}\left\{\log \left(\left|h_{i i}(N)\right|^{2}\right)\right\}}}\right]^{T}\right),
\end{aligned}
$$

and $[.]_{\mathcal{P}_{i}}$ denotes the Euclidean projection on $\mathcal{P}_{i}$. Thus, we can follow the same steps as the proofs in [34, Appendices B and C] to show that the water-filling mapping of our game is a block-contraction under the condition of Theorem 2.

\section{REFERENCES}

[1] A. Pottier, F.-X. Socheleau, and C. Laot, "Distributed Power Allocation Strategy in Shallow Water Acoustic Interference Channels," in IEEE Int. workshop on Signal Processing Advances in Wireless Communications (SPAWC) 2016, Jul. 2016.

[2] A. Pottier, F.-X. Socheleau, and C. Laot, "Adaptive Power Allocation for Noncooperative OFDM Systems in UWA Interference Channels," in 2016 IEEE 3rd Underwater Acoustic Communications and Networking Conference (UComms), Lerici, Sept. 2016, pp. 1-5.

[3] J. Potter, J. Alves, D. Green, G. Zappa, I. Nissen, and K. McCoy, "The State of the Art in Underwater Acoustic Telemetry," Proc. 1st Underwater Communications and Networking Conference (Ucomms), pp. 1-4, Sept. 2014.

[4] R. J. Urick, Principles of Underwater Sound, 3rd Edition, McGraw-Hill, 1983.

[5] N. Baldo, P. Casari, and M. Zorzi, "Cognitive Spectrum Access for Underwater Acoustic Communications," in Proc. MTS/IEEE OCEANS 2008, Quebec City, September 2008.

[6] Z. Wang, S. Zhou, J. Catipovic, and P. Willett, "Parameterized Cancellation of Partial-Band Partial-Block-Duration Interference for Underwater Acoustic OFDM,” IEEE Trans. Signal Process., vol. 60, no. 4, pp. 1782-1795, Apr. 2012.

[7] J. McGee, J. Catipovic, S. Schoenecker, and P. Swaszek, "Leveraging spatial diversity to mitigate partial band interference in undersea networks through waveform reconstruction," in Proc. MTS/IEEE OCEANS 2014 - St John's, Sept 2014, pp. $1-8$.

[8] E. McCarthy, International Regulation Of Underwater Sound: Establishing Rules and Standards to Address Ocean Noise Pollution, Kluwer Academic Publishers, 2004.

[9] S. E. Cho, H. C. Song, and W. S. Hodgkiss, "Successive Interference Cancellation for Underwater Acoustic Communications," IEEE J. Ocean. Eng., vol. 36, no. 4, pp. 490-501, Oct. 2011. 
[10] A. Radosevic, R. Ahmed, T.M. Duman, J. G. Proakis, and M. Stojanovic, “Adaptive OFDM Modulation for Underwater Acoustic Communications: Design Considerations and Experimental Results,” IEEE J. Ocean. Eng., vol. 39, no. 2, pp. 357-370, Apr 2014.

[11] Y. Aval, S. K. Wilson, and M. Stojanovic, "On the Achievable Rate of a Class of Acoustic Channels and Practical Power Allocation Strategies for OFDM Systems,” IEEE J. Ocean. Eng., vol. 40, no. 4, pp. 785-795, Oct 2015.

[12] P. Qarabaqi and M. Stojanovic, "Adaptive Power Control for Underwater Acoustic Communications," in Proc. of IEEE OCEANS 2011, June 2011.

[13] L. Wan, H. Zhou, X. Xu, Y. Huang, S. Zhou, Z. Shi, and J-H. Cui, "Adaptive Modulation and Coding for Underwater Acoustic OFDM," IEEE J. Ocean. Eng., vol. 40, no. 2, pp. 327-336, Apr. 2015.

[14] K. Pelekanakis, L. Cazzanti, G. Zappa, and J. Alves, "Decision Tree-Based Adaptive Modulation for Underwater Acoustic Communications," in 2016 IEEE 3rd Underwater Acoustic Communications and Networking Conference (UComms), Lerici, Sept. 2016, pp. 1-5.

[15] R. Otnes, A. Asterjadhi, P. Casari, M. Goetz, T. Husoy, I. Nissen, K. Rimstad, P. van Walree, and M. Zorzi, Underwater Acoustic Networking Techniques, Springer Briefs in Electrical and Computer Engineering, 2012.

[16] M. Stojanovic, S. Shahabudeen, and M. Chitre, "Underwater Acoustic Communications and Networking: Recent advances and future challenges," Marine Technology Society Journal, vol. 42, no. 1, 2008.

[17] G. Bacci, S. Lasaulce, W. Saad, and L. Sanguinetti, "Game Theory for Networks: A tutorial on game-theoretic tools for emerging signal processing applications," IEEE Signal Processing Magazine, vol. 33, no. 1, pp. 94-119, January 2016.

[18] Z. Han, D. Niyato, W. Saad, T. Basar, and A. Hjorungnes, Game Theory in Wireless and Communication Networks, Cambridge University Press, 2012.

[19] J. F. Nash, “Non Cooperative Games,” The Annals of Mathematics, vol. 54, pp. 286-295, September 1951.

[20] D. Fudenberg and D. K. Levine, The Theory of Learning in Games, Cambridge MA MIT Press, 1998.

[21] F.-X. Socheleau, C. Laot, and J.-M. Passerieux, "Stochastic Replay of non-WSSUS Underwater Acoustic Communication Channels Recorded at Sea," IEEE Trans. Signal Process., vol. 59, no. 10, pp. 4838-4849, 2011.

[22] F-X. Socheleau, C. Laot, and J-M. Passerieux, "Parametric Replay-Based Simulation of Underwater Acoustic Communication Channels," IEEE J. of Oceanic Eng., vol. 40, no. 4, pp. 4838-4839, 2015.

[23] G. Scutari, D.P. Palomar, and S. Barbarossa, "Distributed totally asynchronous iterative waterfilling for wideband interference channel with time/frequency offset," in IEEE Int. Conf. Acoustics, Speech and Signal Processing (ICASSP) 2007, April 2007, vol. 4, pp. IV-1325-IV-1328.

[24] A. Goldsmith, Wireless Communications, Cambridge University Press, 2005.

[25] R. Otnes, P. van Walree, and T. Jenserud, "Validation of Replay-Based Underwater Acoustic Communication Channel Simulation," IEEE J. Ocean. Eng., vol. 38, no. 4, pp. 689-700, 2013.

[26] P. Qarabaqi and M. Stojanovic, "Statistical Characterization and Computationally Efficient Modeling of a Class of Underwater Acoustic Communication Channels," IEEE J. Ocean. Eng., vol. 38, no. 4, pp. 701-717, October 2013.

[27] F.-X. Socheleau, M. Stojanovic, C. Laot, and J.-M. Passerieux, "Information-Theoretic Analysis of Underwater Acoustic OFDM Systems in Highly Dispersive Channels," Journal of Electrical and Computer Engineering, 2012, Article ID 716720.

[28] J.-M. Passerieux, F.-X. Socheleau, and C. Laot, "Achievable Rates over Doubly Selective Rician-Fading Channels under Peak-Power Constraint,” IEEE Trans. Wireless Commun., vol. 12, no. 2, pp. 586-594, February 2013.

[29] D. Fudenberg and J. Tirole, Game Theory, Cambridge MA MIT Press, 1991. 
[30] S. Boyd and L. Vandenberghe, Convex Optimization, Cambridge University Press, 2004.

[31] G. Scutari, D.P. Palomar, and S. Barbarossa, "Asynchronous iterative water-filling for gaussian frequency-selective interference channels," vol. 54, no. 7, pp. 2868-2878, July 2008.

[32] Dimitri P. Bertsekas, "Distributed asynchronous computation of fixed points," Mathematical Programming, vol. 27, no. 1, pp. 107-120, 1983.

[33] G. Scutari, D. P. Palomar, and S. Barbarossa, "Optimal Linear Precoding Strategies for Wideband Non-Cooperative Systems Based on Game Theory-Part I: Nash Equilibria,” IEEE Trans. Signal Process., vol. 56, no. 3, pp. 1230-1249, March 2008.

[34] G. Scutari, D. P. Palomar, and S. Barbarossa, "Optimal Linear Precoding Strategies for Wideband Non-Cooperative Systems Based on Game Theory-Part II: Algorithms,” IEEE Trans. Signal Process., vol. 56, no. 3, pp. 1250-1267, March 2008.

[35] A. Lesherm and E. Zehavi, "Game Theory and the Frequency Selective Interference Channel," IEEE Signal Processing Mag., vol. 26, pp. 28-40, September 2009.

[36] A. J. G. Anandkumar, A. Anandkumar, S. Lambotharan, and J. A. Chambers, "Robust Rate Maximization Game Under Bounded Channel Uncertainty," IEEE Trans. Veh. Technol., vol. 60, no. 9, pp. 4471-4486, Nov 2011.

[37] F-X. Socheleau, C. Laot, and Passerieux J-M., "A Maximum Entropy Framework for Statistical Modeling of Underwater Acoustic Communication Channels," in MTS/IEEE Oceans 2010, Sydney, June 2010, pp. 1-7.

[38] F-X. Socheleau, C. Laot, and J-M. Passerieux, "Concise Derivation of Scattering Function from Channel Entropy Maximization,” IEEE Trans. on Communications, vol. 58, no. 11, pp. 3098-3103, November 2010.

[39] S. Hayashi and Z.Q Luo, "Spectrum Management for Interference-Limited Multiuser Communication Systems," IEEE Trans. Information Theory, vol. 55, no. 3, pp. 1153-1175, March 2009.

[40] P. van Walree, P. Jenserud, and M. Smedsrud, "A Discrete-Time Channel Simulator Driven by Measured Scattering Functions," IEEE J. Sel. Areas Commun., vol. 26, no. 9, pp. 1628-1637, 2008.

[41] F-X. Socheleau, A. Pottier, and C. Laot, "Stochastic Replay of SIMO Underwater Acoustic Communication Channels," OCEANS 2015, pp. 1-6, October 2015.

[42] A. Pottier, F.-X. Socheleau, and C. Laot, "Power-Efficient Spectrum Sharing for Noncooperative Underwater Acoustic Communication Systems,” in MTS/IEEE OCEANS'16 Monterey, Sept. 2016.

[43] S. Ross and B. Chaib-draa, "Satisfaction Equilibrium: Achieving Cooperation in Incomplete Information Games," Proc. Canadian Conference on AI, Quebec City, pp. 61-72, June 2006.

[44] S. Lasaulce M. Debbah S. M. Perlazza, H. Tembine, "Satisfaction Equilibrium: A General Framework for QoS Provisioning in Self-Configuring Networks,” Proc. Global Communications Conference 2010, Miami, Florida, pp. 1-55, Dec. 2010.

[45] A. Lapidoth and S.M. Moser, "Capacity Bounds via Duality with Applications to Multiple-Antenna Systems on Flat-Fading Channels," IEEE Trans. Inform. Theory, vol. 49, no. 10, pp. 2426-2467, October 2003.

[46] W. B. Yang and T. C. Yang, "High-Frequency Channel Characterization for M-ary Frequency Shift Keying Underwater Acoustic Communications," J. Acoust. Soc Am., vol. 120, no. 5, pp. 2615-2626, 2006.

[47] J. C. Preisig and G. B. Deane, "Surface Wave Focusing and Acoustic Communications in the Surf Zone," J. Acoust. Soc. Am., vol. 116, no. 4, pp. 2067-2080, 2004.

[48] C. Tepedelenlioglu, A. Abdi, and G. B. Giannakis, "The Ricean K Factor: Estimation and Performance Analysis," IEEE Trans. on Wireless Comm., vol. 2, no. 4, pp. 799-810, July 2003.

[49] J. B. Rosen, "Existence and Uniqueness of Equilibrium Points for Concave N-Persons Games," Econometrica, vol. 33, no. 3, pp. 521-534, July 1965. 
[50] D.P. Bertsekas and J.N. Tsitsiklis, Parallel and Distributed Computation: Numerical Methods, Belmont, MA: Athena Scientific, 1997.

\begin{tabular}{|l|} 
\\
PLACE \\
PHOTO \\
HERE \\
\hline
\end{tabular}

Antony Pottier received the Dipl.Ing. in electrical engineering from the École Nationale Supérieure des Sciences Appliquées et de Technologie (ENSSAT), Lannion, France in 2013.

From 2014 to 2015, he was a Research Engineer at Telecom Bretagne, Brest, France, where he worked on underwater acoustic communications. He is currently working toward the Ph.D degree at IMT Atlantique (formerly known as Telecom Bretagne), Brest, France. His research interests lie in the field of communications and signal processing and their application to underwater acoustic systems.

\begin{tabular}{|l|}
\hline \\
PLACE \\
PHOTO \\
HERE \\
\end{tabular}

Francois-Xavier Socheleau (S'08-M'12) graduated in electrical engineering from ESEO, Angers, France, in 2001 and received the Ph.D. degree from Telecom Bretagne, Brest, France, in 2011.

From 2001 to 2004, he was a Research Engineer at Thales Communications, France, where he worked on electronic warfare systems. From 2005 to 2007, he was employed at Navman Wireless (New Zealand/U.K.) as an R\&D Engineer. From 2008 to 2011, he worked for Thales Underwater Systems, France. In November 2011, he joined ENSTA Bretagne as an Assistant Professor. Since September 2014, he has been an Associate Professor at IMT Atlantique (formerly known as Telecom Bretagne), France. His research interests include signal processing, wireless communications and underwater acoustics.

Christophe Laot (M'07-SM'12) was born in Brest, France, on March 12, 1967. He received the Eng. degree from the Ecole FRancaise d'Electronique et d'Informatique (EFREI), Paris, France, in 1991 and

PLACE the Ph.D degree from the University of Rennes, France, in 1997.

PHOTO

HERE

In 1997, he joined the Signal and Communications department, IMT Atlantique (formerly known as Telecom Bretagne), Brest, France, as an Associate Professor. Since 2013, he has been (full) Professor in the same institution. His research interests lie in the areas of communications and signal processing, in-

cluding equalization, turbo-equalization, iterative receivers for interference cancellation, synchronization and underwater acoustic communications. Dr C. Laot is member of the IEEE communication society and vice-chair (Europe) of the technology committee "Underwater Communication, Navigation and Positioning" for the IEEE Oceanic Engineering Society. 